\title{
Higgs boson to di-tau channel in chargino-neutralino searches at the LHC
}

\author{
Andreas Papaefstathiou, ${ }^{a}$ Kazuki Sakurai ${ }^{b}$ and Michihisa Takeuchi ${ }^{b}$ \\ ${ }^{a}$ Physik Institut, Universität Zürich, \\ Switzerland \\ ${ }^{b}$ Department of Physics, Theoretical Particle Physics $\&$ Cosmology, \\ King's College London, \\ United Kingdom. \\ E-mail: andreasp@physik.uzh.ch, kazuki.sakurai@kcl.ac.uk, \\ michihisa.takeuchi@kcl.ac.uk
}

ABSTRACT: We consider chargino-neutralino production, $\tilde{\chi}_{2}^{0} \tilde{\chi}_{1}^{ \pm} \rightarrow\left(h \tilde{\chi}_{1}^{0}\right)\left(W^{ \pm} \tilde{\chi}_{1}^{0}\right)$, which results in Higgs boson final states that subsequently decay (inclusively) to leptons (either $h \rightarrow \tau^{+} \tau^{-}$or $\left.h \rightarrow W^{+} W^{-} \rightarrow\left(e^{+} e^{-}, \mu^{+} \mu^{-}, \tau^{+} \tau^{-}\right)+\mathbb{E}_{T}\right)$. Such channels are dominant in large regions of the allowed supersymmetric parameter space for many concrete supersymmetric models. The existence of leptons allows for good control over the backgrounds, rendering this channel competitive to the conventional $h \rightarrow b \bar{b}$ channel. We include hadronic decays of the $\tau$ leptons in our analysis through a $\tau$-identification algorithm. We consider integrated luminosities of $100 \mathrm{fb}^{-1}, 300 \mathrm{fb}^{-1}$ and $3000 \mathrm{fb}^{-1}$, for an LHC running at $p p$ centre-of-mass energy of $14 \mathrm{TeV}$ and provide the expected constraints on the $M_{2}-M_{1}$ plane.

Keywords: Supersymmetry Phenomenology, Hadronic Colliders

ARXIV EPRINT: 1404.1077 


\section{Contents}

1 Introduction 1

2 The $\tilde{\chi}_{2}^{0} \tilde{\chi}_{1}^{ \pm} \rightarrow\left(h \tilde{\chi}_{1}^{0}\right)\left(W^{ \pm} \tilde{\chi}_{1}^{0}\right)$ mode $\quad 4$

2.1 The setup 4

2.2 The cross sections 5

$\begin{array}{lll}2.3 & \text { The branching ratios } & 7\end{array}$

3 Simulation and analysis $\quad \mathbf{8}$

3.1 Monte Carlo simulation 8

$\begin{array}{ll}3.2 \text { Tau identification } & 10\end{array}$

$\begin{array}{lll}3.2 .1 & \text { Tau lepton decay modes } & 10\end{array}$

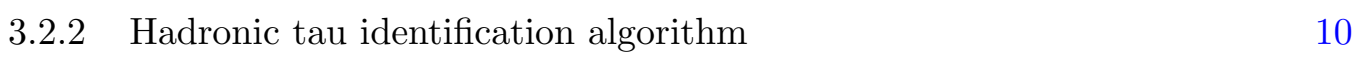

$\begin{array}{lll}3.3 \text { Analysis } & 11\end{array}$

$\begin{array}{lll}3.4 & \text { Signal regions } & 14\end{array}$

$\begin{array}{llr}4 & \text { Results } & 14\end{array}$

5 Conclusions $\quad 16$

$\begin{array}{lr}\text { A Correlation between } \tau \text {-tagging variables } & 18\end{array}$

$\begin{array}{ll}\text { B Definition of the } M_{\min } \text { variable } & 18\end{array}$

$\begin{array}{ll}\text { C Calculation of the initial cross sections } & 21\end{array}$

$\begin{array}{ll}\text { D Discovery with low statistics } & 22\end{array}$

E Cumulative distribution for Poisson random variables 23

$\begin{array}{lll}\text { F Individual signal regions } & 24\end{array}$

\section{Introduction}

One of the primary goals of the CERN Large Hadron Collider (LHC) is to discover or rule out weak scale supersymmetry (SUSY). So far the ATLAS and CMS collaborations have conducted a number of direct SUSY searches in many different channels. The absence of excesses in those channels over the Standard Model (SM) background in turn placed impressive constraints on the SUSY parameter space. The limit is particularly stringent for coloured SUSY particles because of their large production cross sections. For instance, gluino and light flavour squarks are excluded up to masses of about 
$1-1.5 \mathrm{TeV}[1-7]$, although the precise mass bounds depend on the details of the decay chains and mass spectrum. ${ }^{1}$

The recent observation of a SM-like Higgs boson $[12,13]$ also provides interesting implications and opportunities for the exploration of SUSY phenomenology. First of all, the observed mass $\sim 125 \mathrm{GeV}$ and the measured properties of the SM-like Higgs boson are consistent with the lightest CP-even Higgs $(h)$ in the minimal SUSY extension of the SM (MSSM) especially when the masses of scalar superparticles are larger than the several TeV [14-17]. Such scenarios are also consistent with the null results of direct SUSY searches and the precise measurements of flavour-changing neutral currents (FCNC) and CP-violating observables.

Even though the scalars are anticipated to be heavy, it is possible to have relatively light gauginos in the SUSY spectrum. In particular, the electorweak (EW) gauginos can exist and still be very light, since their production cross sections are much smaller than the coloured SUSY particles of the same mass. Indeed, in concrete models, the EW gauginos tend to be much lighter than the coloured SUSY particles. This is due to the fact that the renormalisation group evolution (RGE) increases coloured SUSY particle masses at low energies, owing to their strong QCD interaction, whilst the effect is much smaller for EW gauginos. It is known [18] that if the gaugino GUT relation $\left(M_{3}: M_{2}: M_{1} \sim 7: 2: 1\right)$ holds, the production of EW gauginos can dominate over gluino pair production at the $14 \mathrm{TeV}$ LHC due to the mass hierarchy. Moreover, many SUSY breaking scenarios predict a large mass splitting between gauginos and scalars [19-24]. Unlike the scalar masses, gaugino mass terms are prohibited by R-symmetry, and their mass generation mechanism may be very different. In the scenarios where R-symmetry is only weakly broken, the gauginos tend to be much lighter than the scalars. In such scenarios, gauginos are the only SUSY particles which are accessible at the LHC [25-28].

The EW gauginos, namely, charginos and neutralinos, have already been intensively searched for at the LHC. ATLAS and CMS interpreted their results in the context of simplified models, where several assumptions were made. For instance, the lightest neutralino $\left(\tilde{\chi}_{1}^{0}\right)$ was assumed to be bino-like and the second lightest neutralino $\left(\tilde{\chi}_{2}^{0}\right)$ and the lighter chargino $\left(\tilde{\chi}_{1}^{ \pm}\right)$wino-like, while possessing the same mass, $m_{\tilde{\chi}_{2}^{0}}=m_{\tilde{\chi}_{1}^{ \pm}}$. In these simplified models, particular decays of the chargino and the second lightest neutralino with $100 \%$ branching ratios were considered. The most stringent constraints were found for the models where the $\tilde{\chi}_{2}^{0}$ and $\tilde{\chi}_{1}^{ \pm}$decay exclusively into on-shell sleptons $(\tilde{\ell}$ and $\tilde{\nu})$. In this case, $m_{\tilde{\chi}_{2}^{0}}=m_{\tilde{\chi}_{1}^{ \pm}}$is excluded up to about $700 \mathrm{GeV}$ with $m_{\tilde{\chi}_{1}^{0}} \lesssim 300 \mathrm{GeV}[29,30]$. Simplified models with the chargino and neutralino decays leading to di- $\tau$ final states via on-shell $\tilde{\tau}$ and $\tilde{\nu}_{\tau}$ have also been searched for, and the limit was found to be $m_{\tilde{\chi}_{2}^{0}}=m_{\tilde{\chi}_{1}^{ \pm}} \gtrsim 300$ (350) $\mathrm{GeV}$ with $m_{\tilde{\chi}_{1}^{0}} \lesssim 100(50) \mathrm{GeV}[29,30]$. If the sleptons and staus are heavier than the EW gauginos, the $\tilde{\chi}_{1}^{ \pm}$predominantly decays to $W^{ \pm}$and $\tilde{\chi}_{1}^{0}$. On the other hand, the $\tilde{\chi}_{2}^{0}$ has two possible decay modes: $\tilde{\chi}_{2}^{0} \rightarrow Z \tilde{\chi}_{1}^{0}$ and $\tilde{\chi}_{2}^{0} \rightarrow h \tilde{\chi}_{1}^{0}$. The former has been searched for and the resulting limit was $m_{\tilde{\chi}_{2}^{0}}=m_{\tilde{\chi}_{1}^{ \pm}} \gtrsim 350$ with $m_{\tilde{\chi}_{1}^{0}} \lesssim 100 \mathrm{GeV}$ [29]. The latter process, $\tilde{\chi}_{2}^{0} \tilde{\chi}_{1}^{ \pm} \rightarrow\left(h \tilde{\chi}_{1}^{0}\right)\left(W^{ \pm} \tilde{\chi}_{1}^{0}\right)$, has also been looked for recently by employing the $h \rightarrow b \bar{b}$ channel.

\footnotetext{
${ }^{1}$ See, e.g., $[8-11]$ for the recent ideas and programmes to address this problem.
} 


\begin{tabular}{|ll|}
\hline lower bound on $m_{\tilde{\chi}_{2}^{0}}=m_{\tilde{\chi}_{1}^{ \pm}}$ & search hypothesis \\
\hline $700 \mathrm{GeV}[29,30]$ & $\tilde{\chi}_{2}^{0}, \tilde{\chi}_{1}^{ \pm}$both decay exclusively to \\
& sleptons, $m_{\tilde{\chi}_{1}^{0}}=300 \mathrm{GeV}$. \\
$300(350) \mathrm{GeV}[29,30]$ & $m_{\tilde{\chi}_{1}^{0} \lesssim 100(50) \mathrm{GeV} .}$ \\
$350 \mathrm{GeV}[29]$ & staus heavier than EW gauginos, \\
& $\tilde{\chi}_{1}^{ \pm} \rightarrow W^{ \pm} \tilde{\chi}_{2}^{0}, \tilde{\chi}_{2}^{0} \rightarrow Z \tilde{\chi}_{1}^{0}, m_{\tilde{\chi}_{1}^{0}} \lesssim$ \\
& $100 \mathrm{GeV}$. \\
$200 \mathrm{GeV}[31], 300 \mathrm{GeV}[32]$ & staus heavier than EW gauginos, \\
& $\tilde{\chi}_{1}^{ \pm} \rightarrow W^{ \pm} \tilde{\chi}_{2}^{0}, \tilde{\chi}_{1}^{ \pm} \rightarrow h \tilde{\chi}_{1}^{0}$, with \\
& $h \rightarrow b \bar{b}$, when $m_{\tilde{\chi}_{1}^{0}} \lesssim 30 \mathrm{GeV}$. \\
\hline
\end{tabular}

Table 1. Summary of the EW gaugino searches as given in the main text.

This channel suffers from an overwhelmingly large $t \bar{t}$ background and only weak constraints have been found. The bound is $m_{\tilde{\chi}_{2}^{0}}=m_{\tilde{\chi}_{1}^{ \pm}} \gtrsim 200 \mathrm{GeV}$ [31] and $300 \mathrm{GeV}$ [32] only when $m_{\tilde{\chi}_{1}^{0}} \lesssim 30 \mathrm{GeV}$. A summary of EW gaugino searches is given in Table 1 for convenience.

The fact that current searches provide weak constraints is not the only reason the $\tilde{\chi}_{2}^{0} \tilde{\chi}_{1}^{ \pm} \rightarrow\left(h \tilde{\chi}_{1}^{0}\right)\left(W^{ \pm} \tilde{\chi}_{1}^{0}\right)$ process is especially interesting for further study. Firstly, in this process one can take advantage of the discovery of the SM-like Higgs boson, making use of the of its properties as measured in the present dataset [33]. Identifying the observed boson as the lightest CP-even Higgs in the MSSM allows us to make a precise prediction of the $\tilde{\chi}_{2}^{0} \tilde{\chi}_{1}^{ \pm} \rightarrow\left(h \tilde{\chi}_{1}^{0}\right)\left(W^{ \pm} \tilde{\chi}_{1}^{0}\right)$ signature, which is necessary for the limit calculation and also useful in designing optimal search strategies for this mode. Secondly, as we will see in section 2 , the scenarios with heavy scalar SUSY particles may imply that $\tilde{\chi}_{2}^{0}$ predominantly decays into $h$ and $\tilde{\chi}_{1}^{0}$.

In this paper, we study the exclusion and discovery reach of the $\tilde{\chi}_{2}^{0} \tilde{\chi}_{1}^{ \pm} \rightarrow\left(h \tilde{\chi}_{1}^{0}\right)\left(W^{ \pm} \tilde{\chi}_{1}^{0}\right)$ process, using the $W$ decays to electrons muons or taus and the $h \rightarrow \tau \tau$ and $h \rightarrow W W \rightarrow$ $(\tau / \ell, \nu)(\tau / \ell, \nu)$ modes. Our study differs from earlier studies for $\tilde{\chi}_{2}^{0} \tilde{\chi}_{1}^{ \pm} \rightarrow\left(h \tilde{\chi}_{1}^{0}\right)\left(W^{ \pm} \tilde{\chi}_{1}^{0}\right)[18$, 33-35], which have focused on the decays of the $W$ to electrons or muons and $h \rightarrow b \bar{b}$ modes. The obvious advantage of the channel with $h \rightarrow b \bar{b}$ is its relatively large branching ratio $\mathrm{BR}(h \rightarrow b \bar{b})$. However, this channel suffers from an overwhelming $t \bar{t}$ background. Employing $h \rightarrow \tau \tau$ and $h \rightarrow W W \rightarrow(\tau / \ell, \nu)(\tau / \ell, \nu)$ introduces a reduction of the branching ratio, by a factor of $[B R(h \rightarrow \tau \bar{\tau})+B R(h \rightarrow W W \rightarrow(\tau / \ell, \nu)(\tau / \ell, \nu))] / B R(h \rightarrow b \bar{b}) \sim 0.15$, but the $t \bar{t}$ background can be reduced significantly by vetoing $b$-jets and requiring two $\tau \mathrm{s}$ in the final state as we will see in section 3 . We will demonstrate that the channel with $h \rightarrow \tau \tau$ and $h \rightarrow W W \rightarrow(\tau / \ell, \nu)(\tau / \ell, \nu)$ can provide competitive discovery and exclusion prospects to those obtained in the channel with the $h \rightarrow b \bar{b}$ mode.

Revealing the details of the EW gaugino sector is especially important. It is commonly believed that this sector contains the particle that can be a candidate for dark matter. Moreover, studying the accessible mass scale of the EW gauginos at the LHC is important [36] for the planning of future collider programmes. 
The article is organised as follows: in the next section, we provide the details of the setup we use for the $\tilde{\chi}_{2}^{0} \tilde{\chi}_{1}^{ \pm} \rightarrow\left(h \tilde{\chi}_{1}^{0}\right)\left(W^{ \pm} \tilde{\chi}_{1}^{0}\right)$ mode and discuss the cross section and branching ratio of EW gauginos with particular attention to heavy scalar scenarios with a large $\mu$-term. In section 3 we provide details of the Monte Carlo simulation performed to generate the samples used in the analysis and give details of the algorithm employed for the identification of jets originating from hadronic decays of $\tau$ leptons. We then proceed to outline the details of our discrimination analysis, which forms the basis for defining the signal regions for the SUSY parameter space scan. The results of the parameter space scan are presented and discussed in section 4 . We conclude in section 5. Supplementary appendices describe the definition of a kinematic variable used in our analysis, the calculation of cross sections for signal and background and statistical methods with low event numbers. The last appendix in particular describes a systematic way to recast our results onto the other scenarios. The application includes higgsino NSLP scenarios with a bino LSP and higgsino/wino NLSP scenarios with a gravitino LSP as discussed, for example, in [37-40].

\section{The $\tilde{\chi}_{2}^{0} \tilde{\chi}_{1}^{ \pm} \rightarrow\left(h \tilde{\chi}_{1}^{0}\right)\left(W^{ \pm} \tilde{\chi}_{1}^{0}\right)$ mode}

In this section we describe the setup of our analysis and clarify the assumptions we made in the chargino and neutralino sectors. Moreover, we discuss the cross sections and branching ratios of the production and decay modes relevant to our analysis.

\subsection{The setup}

Throughout this paper we consider CP-conserving EW gaugino sector and assume $m_{\tilde{\chi}_{2}^{0}} \simeq$ $m_{\tilde{\chi}_{1}^{ \pm}}>m_{\tilde{\chi}_{1}^{0}}$ for simplicity. This relation is realised in many SUSY breaking scenarios, particularly in the cases where $|\mu| \gg M_{2}>M_{1}$ and $M_{2} \gg|\mu|>M_{1}$. The former case is motivated by the heavy scalar scenario. In the MSSM, the soft scalar masses for $H_{u}$ and $H_{d}$ and the $\mu$-parameter are related by the EW symmetry breaking condition [41]

$$
\frac{m_{Z}^{2}}{2}=\frac{m_{H_{d}}^{2}-m_{H_{u}}^{2} \tan ^{2} \beta}{\tan ^{2} \beta-1}-|\mu|^{2} .
$$

In the heavy scalar scenario, the scale of $m_{H_{u}}$ and $m_{H_{d}}$ is very large $\left(m_{H_{u} / d} \gg M_{2}\right)$. In this case, the condition (2.1) implies that the $\mu$-parameter is expected to be of the same scale as the scalar masses, unless $m_{H_{u}}$ and $m_{H_{d}}$ are carefully tuned at the EW scale in such a way that the first terms in the right hand side of eq. (2.1) becomes unnaturally small. ${ }^{2}$

In this section we assume the scale of $\mu$ is equal to the scalar masses and $|\mu| \gg M_{2}>$ $M_{1}>0$. However, the collider analysis described in section 3 is applicable to other scenarios as far as the $\tilde{N} \tilde{C}^{ \pm} \rightarrow(h \chi)\left(W^{ \pm} \chi\right)$ topology is concerned, where $\tilde{N}$ and $\tilde{C}^{ \pm}$are massive BSM particles with the same mass and $\chi$ is an invisible particle with an arbitrary mass. One such scenario involves a bino LSP scenario with a higgsino NLSP, $M_{2} \gg|\mu|>M_{1}$. The application also includes gravitino LSP scenarios with wino or higgsino NSLP as discussed for example in [37-40], where the same topology is realised by $\tilde{\chi}_{1}^{0} \tilde{\chi}_{1}^{ \pm} \rightarrow(h \tilde{G})\left(W^{ \pm} \tilde{G}\right)$ with $\tilde{G}$ being gravitino. We will get back to this point in the end of this section.

\footnotetext{
${ }^{2}$ Even in that case, the same size of tuning is required on the $\mu$-parameter.
} 


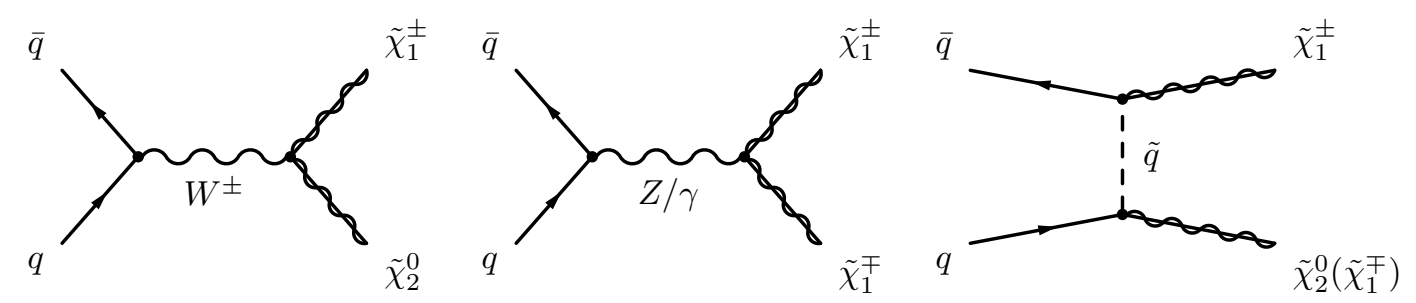

Figure 1. The tree-level diagrams for the relevant $\tilde{\chi}_{2}^{0}$ and $\tilde{\chi}_{1}^{ \pm}$production.

\subsection{The cross sections}

Figure 1 shows the tree-level diagrams for the relevant modes of $\tilde{\chi}_{2}^{0}$ and $\tilde{\chi}_{1}^{ \pm}$production. There are two types of diagrams which may interfere: $s$-channel diagrams with gauge boson exchange and $t$-channel diagrams with squark exchange. The $t$-channel diagrams are suppressed by the squark mass and it is expected that the contribution of this diagram decreases as the squark mass increases.

Figure 2 shows the NLO production cross sections for the $\tilde{\chi}_{2}^{0} \tilde{\chi}_{1}^{ \pm}$and $\tilde{\chi}_{1}^{+} \tilde{\chi}_{1}^{-}$modes at the $14 \mathrm{TeV}$ LHC as functions of the squark mass. The cross sections have been calculated using Prospino $2.1[42,43]$ with all the charges summed. In the plot and throughout the paper, we take $|\mu|=m_{\tilde{q}}$ for simplicity. For the specific plot, we take $M_{2}=350 \mathrm{GeV}$ and $M_{1}=100 \mathrm{GeV}$. The solid and dashed curves correspond to $\tan \beta=2$ and 50 , respectively. As a result of destructive interference between the $s$-channel gauge boson exchange diagram and the $t$-channel squark exchange diagram, the $\tilde{\chi}_{2}^{0} \tilde{\chi}_{1}^{ \pm}$and $\tilde{\chi}_{1}^{+} \tilde{\chi}_{1}^{-}$production cross sections increase as the squark mass increases. For a squark mass larger than $\sim 4 \mathrm{TeV}$, the contribution of the squark exchange diagram is decoupled and the cross sections become insensitive to the squark mass. It is interesting to note that the $\tilde{\chi}_{2}^{0} \tilde{\chi}_{1}^{ \pm}$and $\tilde{\chi}_{1}^{+} \tilde{\chi}_{1}^{-}$cross sections are maximised in the limit of large squark mass. This gives additional motivation to perform EW gaugino searches in the context of heavy scalar scenarios.

Figure 3 shows the NLO cross sections for various gaugino production modes at the $14 \mathrm{TeV}$ LHC. We have assumed the gaugino GUT relation, $M_{3}: M_{2}: M_{1}=7: 2: 1$, at the EW scale and plotted the cross sections as functions of $M_{2}$ (and $m_{\tilde{g}} \simeq M_{3}=7 M_{2} / 2$ ). The other relevant parameters were fixed as $m_{\tilde{q}}=\mu=3 \mathrm{TeV}$ and $\tan \beta=10$.

One can see that the $\tilde{\chi}_{2}^{0} \tilde{\chi}_{1}^{ \pm}$and $\tilde{\chi}_{1}^{+} \tilde{\chi}_{1}^{-}$production modes have substantial cross sections. Because of the large mass hierarchy in the gaugino GUT relation, the $\tilde{g} \tilde{g}$ cross section drops much faster than the EW gaugino production cross sections as $M_{2}$ increases. Due to this effect, $\tilde{\chi}_{2}^{0} \tilde{\chi}_{1}^{ \pm}$and $\tilde{\chi}_{1}^{+} \tilde{\chi}_{1}^{-}$production dominate over $\tilde{g} \tilde{g}$ production for $M_{2} \gtrsim 350 \mathrm{GeV}$.

The EW gaugino production modes other than $\tilde{\chi}_{2}^{0} \tilde{\chi}_{1}^{ \pm}$and $\tilde{\chi}_{1}^{+} \tilde{\chi}_{1}^{-}$have cross sections which are a few orders of magnitude smaller. This is due to the fact that these production modes contain at least one bino state or two $\tilde{W}^{0}$ states in the large $\mu$ limit, and there exists no gaugino-gaugino-gauge boson couplings for those states.

As can be seen from figures 2 and 3 , the $\tilde{\chi}_{2}^{0} \tilde{\chi}_{1}^{ \pm}$cross section is more than two times larger than the $\tilde{\chi}_{1}^{+} \tilde{\chi}_{1}^{-}$cross section. This is mainly because $\tilde{\chi}_{2}^{0} \tilde{\chi}_{1}^{ \pm}$contains two distinctive modes: $\tilde{\chi}_{2}^{0} \tilde{\chi}_{1}^{+}$with $W^{+}$exchange and $\tilde{\chi}_{2}^{0} \tilde{\chi}_{1}^{-}$with $W^{-}$exchange. It is therefore more beneficial to target the $\tilde{\chi}_{2}^{0} \tilde{\chi}_{1}^{ \pm}$production mode in the EW gaugino searches. 


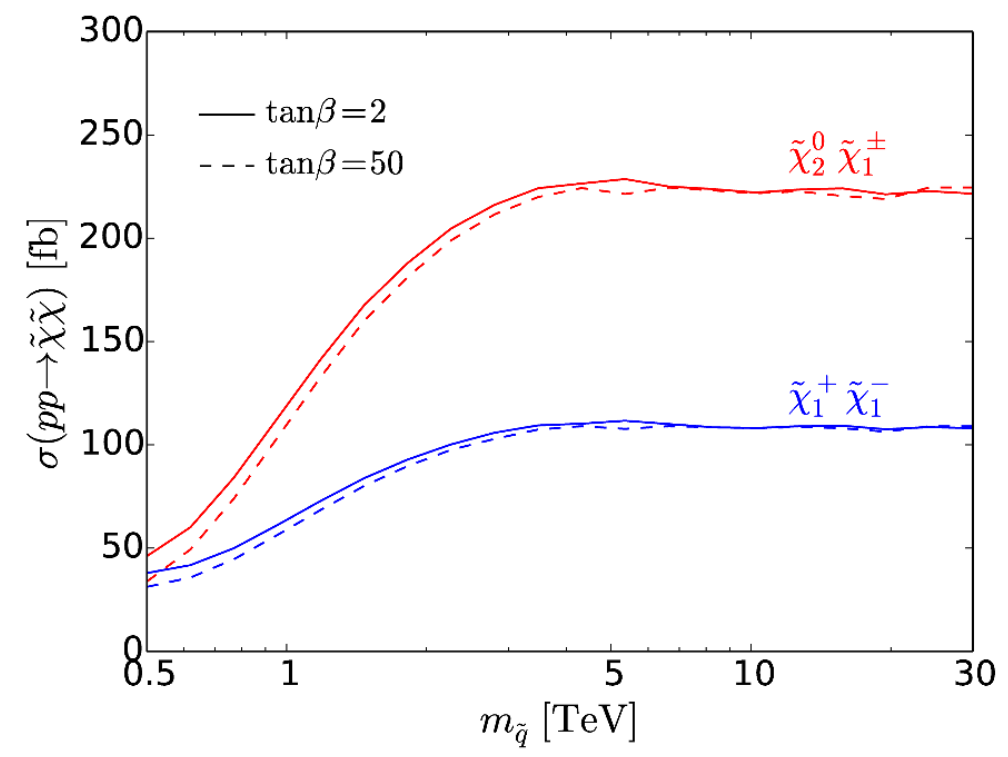

Figure 2. The NLO production cross sections for the $\tilde{\chi}_{1}^{0} \tilde{\chi}_{1}^{ \pm}$and $\tilde{\chi}_{1}^{+} \tilde{\chi}_{1}^{-}$modes at the $14 \mathrm{TeV}$ LHC as functions of the squark mass. The cross sections have been calculated using Prospino 2.1 [42, 43] with all the charges summed. We have set $\mu=m_{\tilde{q}}$ and $M_{2}=350 \mathrm{GeV}$ and $M_{1}=100 \mathrm{GeV}$. The solid and dashed curves correspond to $\tan \beta=2$ and 50, respectively.

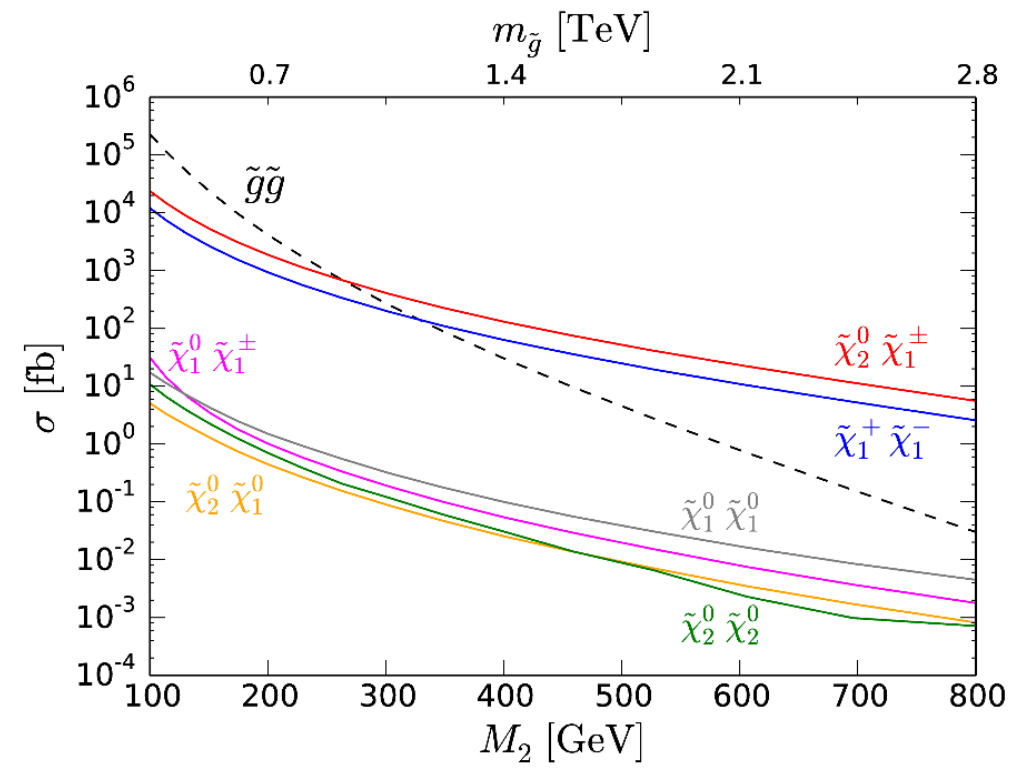

Figure 3. The NLO cross sections for various gaugino production modes at the $14 \mathrm{TeV} \mathrm{LHC}$ as functions of $M_{2}$ (and $m_{\tilde{g}} \simeq M_{3}=7 M_{2} / 2$ ). We have assumed the gaugino GUT relation, $M_{3}: M_{2}: M_{1}=7: 2: 1$, at the EW scale. The other relevant parameters were fixed as $m_{\tilde{q}}=\mu=3 \mathrm{TeV}$ and $\tan \beta=10$. 

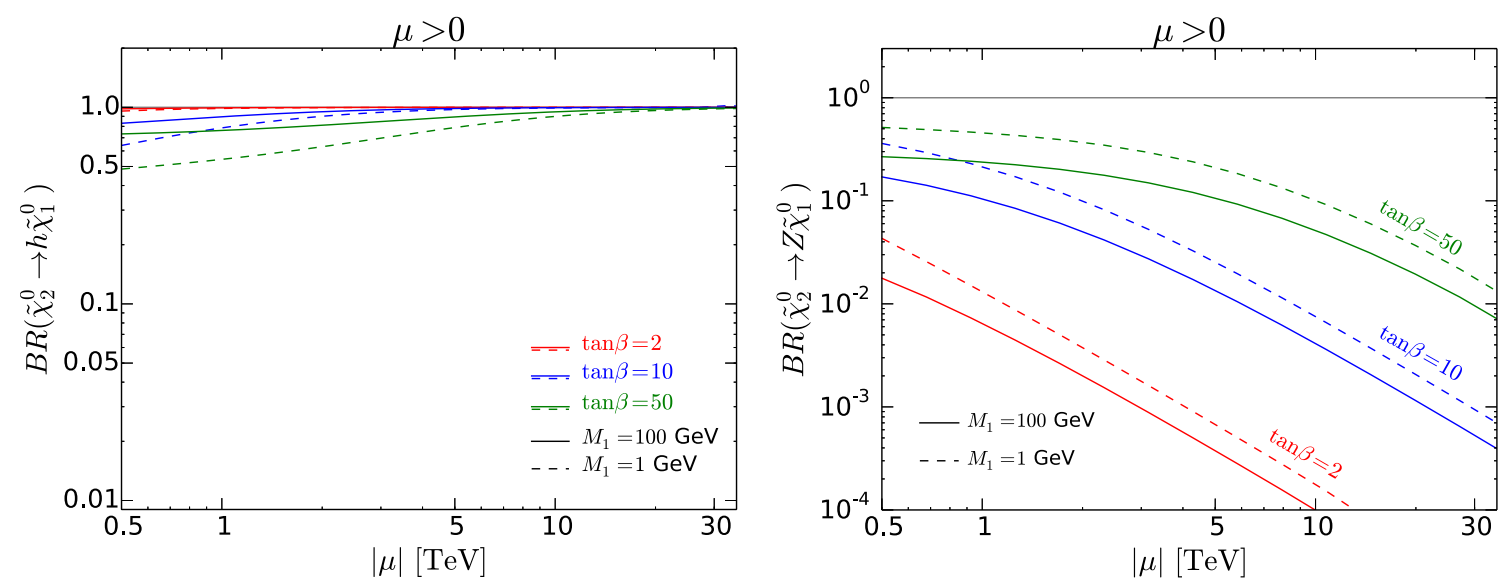

Figure 4. The branching ratios of $\tilde{\chi}_{2}^{0} \rightarrow h \tilde{\chi}_{1}^{0}$ (left) and $\tilde{\chi}_{2}^{0} \rightarrow Z \tilde{\chi}_{1}^{0}$ (right) modes as functions of $|\mu|$ in the $\mu>0$ case. $M_{2}$ is taken to be $350 \mathrm{GeV}$ and $m_{h}=125.5 \mathrm{GeV}$. We have fixed $M_{2}=350 \mathrm{GeV}$ but we show variations of $\tan \beta$ and $M_{1}$ as $\tan \beta=2$ (red), 10 (blue), 50 (green) and $M_{1}=100 \mathrm{GeV}$ (solid), $1 \mathrm{GeV}$ (dashed).

\subsection{The branching ratios}

If scalar fermions and the MSSM Higgs bosons (other than the SM-like one) are heavier than the $\tilde{\chi}_{1}^{ \pm}$and $\tilde{\chi}_{2}^{0}$, these gaugino states decay predominantly into $\tilde{\chi}_{1}^{0}$ and SM bosons, $W^{ \pm}, Z$ and $h$, if the decays are kinematically allowed. In this case, $\tilde{\chi}_{1}^{ \pm}$exclusively decays into $W^{ \pm}$and $\tilde{\chi}_{1}^{0}$ with $\mathrm{BR} \sim 100 \%$. On the other hand, $\tilde{\chi}_{2}^{0}$ has two possible decay modes: $\tilde{\chi}_{2}^{0} \rightarrow Z \tilde{\chi}_{1}^{0}$ and $\tilde{\chi}_{2}^{0} \rightarrow h \tilde{\chi}_{1}^{0}$. The decay rates of these modes are determined by the $\tilde{\chi}_{2}^{0} \tilde{\chi}_{1}^{0} Z / h$ couplings, up to the phase space factor and the polarisation effect. In the limit of large $|\mu|$ and heavy MSSM Higgs bosons, the $\tilde{\chi}_{2}^{0} \tilde{\chi}_{1}^{0} Z / h$ couplings in the CP-conserving case are given by $[44,45]$

$$
\begin{aligned}
\left|C_{\tilde{\chi}_{1}^{0} \tilde{\chi}_{2}^{0} Z}\right| & \simeq \frac{e}{2} \frac{m_{Z}^{2}}{|\mu|^{2}} \\
\left|C_{\tilde{\chi}_{1}^{0} \tilde{\chi}_{2}^{0} h}\right| & \simeq \frac{e}{2} \frac{m_{Z}}{|\mu|}\left|2 \sin 2 \beta+\frac{M_{1}+M_{2}}{\mu}\right|,
\end{aligned}
$$

where $e$ is the electric charge $\left(\alpha_{e m}=e^{2} /(4 \pi)\right)$.

Figure 4 shows the branching ratios of $\tilde{\chi}_{2}^{0} \rightarrow h \tilde{\chi}_{1}^{0}$ (left) and $\tilde{\chi}_{2}^{0} \rightarrow Z \tilde{\chi}_{1}^{0}$ (right) modes as functions of $|\mu|$ in the $\mu>0$ case. $M_{2}$ has been fixed to $M_{2}=350 \mathrm{GeV}$ but $\tan \beta$ and $M_{1}$ are varied as $\tan \beta=2$ (red), 10 (blue), 50 (green) and $M_{1}=100 \mathrm{GeV}$ (solid), $1 \mathrm{GeV}$ (dashed). Here and throughout the paper, we have explicitly set $m_{h}=125.5 \mathrm{GeV}$. This condition can be always realised by tuning the stop mass, which has no effect on the EW gaugino sector, and hence on our phenomenological analysis. The branching ratios were calculated using SUSY-HIT [46].

One can see that the $\tilde{\chi}_{2}^{0} \rightarrow h \tilde{\chi}_{1}^{0}$ mode is enhanced, whilst the $\tilde{\chi}_{2}^{0} \rightarrow Z \tilde{\chi}_{1}^{0}$ mode is suppressed as $|\mu|$ increases. This is due to the $\tilde{\chi}_{1}^{0} \tilde{\chi}_{2}^{0} Z$ coupling having the extra $m_{Z} / \mu$ suppression factor compared to the $\tilde{\chi}_{1}^{0} \tilde{\chi}_{2}^{0} h$ coupling, as seen in eq. (2.2). In the $|\mu| \gtrsim$ $500 \mathrm{GeV}$ region, the $\tilde{\chi}_{2}^{0} \rightarrow h \tilde{\chi}_{1}^{0}$ mode has $\mathrm{BR} \gtrsim 60 \%$ and dominates the $\tilde{\chi}_{2}^{0}$ decay, apart from 
the $\tan \beta=50, M_{1}=1 \mathrm{GeV}$ case. For moderate values of $\mu, 0.5 \lesssim \mu / \mathrm{TeV} \lesssim 3$, the factor $\left|2 \sin 2 \beta+\left(M_{1}+M_{2}\right) / \mu\right|$ in $C_{\tilde{\chi}_{1}^{0} \tilde{\chi}_{2}^{0} h}$ is important in the competition between the $\tilde{\chi}_{2}^{0} \rightarrow h \tilde{\chi}_{1}^{0}$ and $\tilde{\chi}_{2}^{0} \rightarrow Z \tilde{\chi}_{1}^{0}$ modes and at $\mu \sim 1 \mathrm{TeV}, \tan \beta \sim 50, M_{1} \sim 1 \mathrm{GeV}, B R\left(\tilde{\chi}_{2}^{0} \rightarrow Z \tilde{\chi}_{1}^{0}\right)$ can be as large as $B R\left(\tilde{\chi}_{2}^{0} \rightarrow h \tilde{\chi}_{1}^{0}\right)$. However, in the large $|\mu|$ limit $B R\left(\tilde{\chi}_{2}^{0} \rightarrow h \tilde{\chi}_{1}^{0}\right)$ approaches $100 \%$ independently of $\tan \beta$ and $M_{1}$ as long as the phase space is open.

Figure 5 is equivalent to figure 4 , with $\mu$ instead set to $\mu<0$. One can see that $B R\left(\tilde{\chi}_{2}^{0} \rightarrow h \tilde{\chi}_{1}^{0}\right)$ becomes zero at a particular $|\mu|$ value depending on $\tan \beta$ and $M_{1}$. This is due to the cancellation between the two terms in the $\left|2 \sin 2 \beta+\left(M_{1}+M_{2}\right) / \mu\right|$ factor in the $\tilde{\chi}_{1}^{0} \tilde{\chi}_{2}^{0} h$ coupling. As can be seen, this cancellation occurs at $\mu \sim-1 \mathrm{TeV}$ for $\tan \beta \sim 10$ and $\mu \sim-5 \mathrm{TeV}$ for $\tan \beta \sim 50$. As $|\mu|$ increases, $B R\left(\tilde{\chi}_{2}^{0} \rightarrow h \tilde{\chi}_{1}^{0}\right)$ quickly approaches $100 \%$ following the cancellation. For $|\mu| \gtrsim 10 \mathrm{TeV}$, the $\tilde{\chi}_{2}^{0} \rightarrow h \tilde{\chi}_{1}^{0}$ mode dominates over the $\tilde{\chi}_{2}^{0}$ decay, independently of $\tan \beta$ and $M_{1}$.

To summarise, we have demonstrated that in the scenarios with large $m_{\tilde{q}}$ and $|\mu|, \tilde{\chi}_{2}^{0}$ and $\tilde{\chi}_{1}^{ \pm}$become wino-like gauginos with $m_{\tilde{\chi}_{2}^{0}} \simeq m_{\tilde{\chi}_{1}^{ \pm}} \simeq M_{2}$ and $\tilde{\chi}_{2}^{0} \tilde{\chi}_{1}^{ \pm}$has the largest cross section among the EW gaugino production modes. We also argued that in such scenarios $\tilde{\chi}_{1}^{ \pm}$predominantly decays into $W^{ \pm}$and $\tilde{\chi}_{1}^{0}$ and the $\tilde{\chi}_{2}^{0} \rightarrow h \tilde{\chi}_{1}^{0}$ mode typically dominates the $\tilde{\chi}_{2}^{0}$ decay. These arguments provide a strong motivation to study the $p p \rightarrow \tilde{\chi}_{2}^{0} \tilde{\chi}_{1}^{ \pm} \rightarrow$ $\left(h \tilde{\chi}_{1}^{0}\right)\left(W^{ \pm} \tilde{\chi}_{1}^{0}\right)$ mode in the EW gaugino searches in the scenarios with large $m_{\tilde{q}}$ and $|\mu|$.

In the following sections, we study the $p p \rightarrow \tilde{\chi}_{2}^{0} \tilde{\chi}_{1}^{ \pm} \rightarrow\left(h \tilde{\chi}_{1}^{0}\right)\left(W^{ \pm} \tilde{\chi}_{1}^{0}\right)$ channel using the $W \rightarrow \tau / \ell, \nu$ plus $h \rightarrow \tau \tau$ and $h \rightarrow W W \rightarrow(\tau / \ell, \nu)(\tau / \ell, \nu)$ channel. We set $m_{\tilde{q}}=$ $\mu=m_{A}=3 \mathrm{TeV}, \tan \beta=10$ throughout. This leads to $B R\left(\tilde{\chi}_{1}^{ \pm} \rightarrow W^{ \pm} \tilde{\chi}_{1}^{0}\right) \simeq B R\left(\tilde{\chi}_{2}^{0} \rightarrow\right.$ $\left.h \tilde{\chi}_{1}^{0}\right) \simeq 100 \%$. With this parameter choice, the lightest CP-even Higgs becomes SM-like and we use the same branching ratios as those for the SM Higgs boson. Although the above parameter set is motivated by the heavy scalar scenario, our analysis can easily be recast onto other SUSY scenarios. Changing the above parameters may modify the $p p \rightarrow \tilde{\chi}_{2}^{0} \tilde{\chi}_{1}^{ \pm}$ cross section and the $\tilde{\chi}_{2}^{0} \rightarrow h \tilde{\chi}_{1}^{0}$ branching ratio significantly but does not alter the signal efficiencies for the signal regions defined in the next section. The discovery reach and exclusion limit for a different set of parameters can therefore be obtained by rescaling the cross section and branching ratio accordingly. Moreover, the calculated signal efficiencies can also be used for a larger class of models as far as the $\tilde{N} \tilde{C}^{ \pm} \rightarrow(h \chi)\left(W^{ \pm} \chi\right)$ topology is concerned, as mentioned in subsection 2.1. Neglecting a finite width effect and spin correlations, the signal efficiencies will be very similar between $\tilde{\chi}_{2}^{0} \tilde{\chi}_{1}^{ \pm} \rightarrow\left(h \tilde{\chi}_{1}^{0}\right)\left(W^{ \pm} \tilde{\chi}_{1}^{0}\right)$ and $\tilde{N} \tilde{C}^{ \pm} \rightarrow(h \chi)\left(W^{ \pm} \chi\right)$ at $\left(m_{\tilde{\chi}_{2}^{0}}=m_{\tilde{\chi}_{1}^{ \pm}}, m_{\tilde{\chi}_{1}^{0}}\right)=\left(m_{\tilde{N}}=m_{\tilde{C}}, m_{\chi}\right)$. We explain this point in more detail in appendix $\mathrm{F}$ and provide the necessary information to perform such a re-analysis.

\section{Simulation and analysis}

\subsection{Monte Carlo simulation}

The SUSY $p p \rightarrow \tilde{\chi}_{2}^{0} \tilde{\chi}_{1}^{ \pm} \rightarrow\left(h \tilde{\chi}_{1}^{0}\right)\left(W^{ \pm} \tilde{\chi}_{1}^{0}\right)$ signals were generated using the HERWIG ++ general-purpose event generator [47-49] via SUSY Les Houches Accord files used as input for the parameter points, according to the assumptions outlined in the previous section. The signal cross sections were scaled to the next-to-leading order cross sections using results 

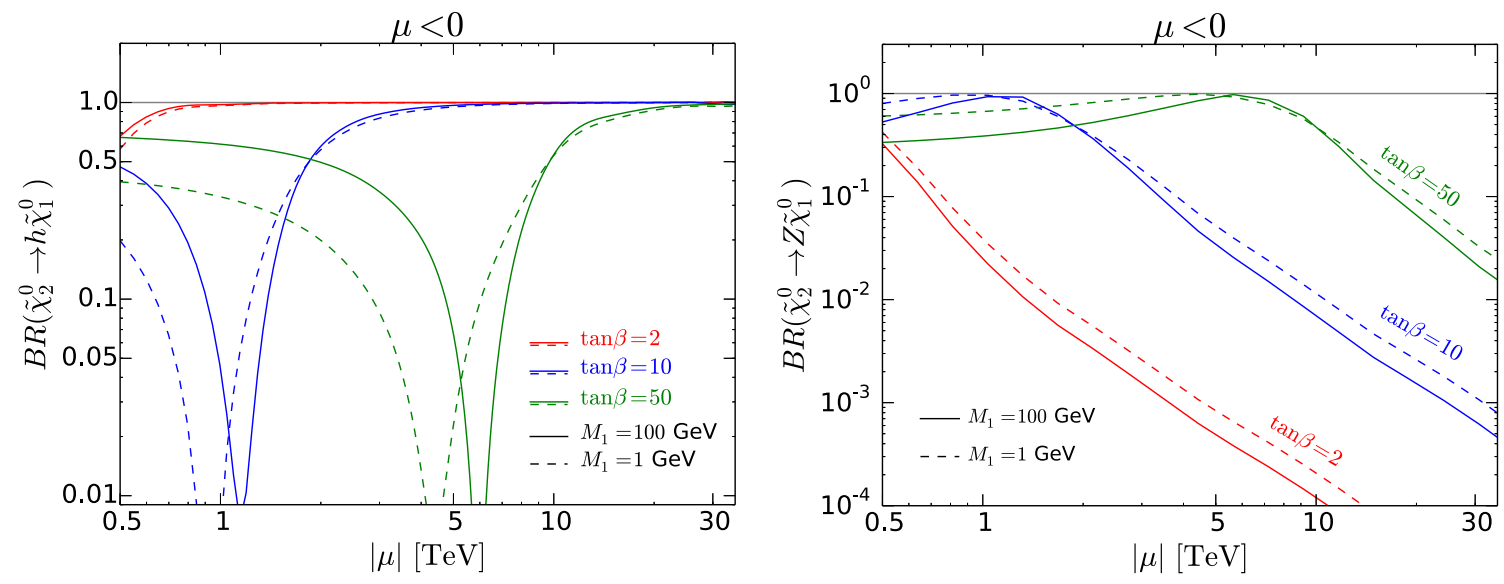

Figure 5. Equivalent plots to figure 4 but with $\mu<0$.

obtained from Prospino 2.1. The $h V, t \bar{t}, t \bar{t} h$ and $W Z$ backgrounds were also generated internally in HERWIG ++ at leading order. The $Z+$ jets and $W+$ jets backgrounds were generated using the parton-level matrix element generator AlpGen and merged with the HERWIG ++ parton shower using the MLM method [50-52]. The generator-level cuts on the $V+$ jets backgrounds were taken to be $p_{T j, \min }=15 \mathrm{GeV}, \eta_{j, \max }=3.0, \Delta R_{j, \min }=0.2$ with $m_{\ell \ell} \in(15,160) \mathrm{GeV}$ (or $m_{\tau, \tau}$ ) for $V=Z$. For the $Z+$ jets case we considered matrix elements with one extra parton merged to the shower, whereas for the $W+$ jets case we considered matrix elements with two partons merged to the shower.

For the signal we allowed the $W$ to decay to all lepton flavours, including taus. Likewise, for the backgrounds we consider all of the leptonic decays of the $W$ and $Z$, to muons, electrons or taus. We consider the Monte Carlo samples of the $Z$ and $W$ backgrounds going to electrons or muons separately from those going to taus, as they would have different amounts of missing energy, leptons and jets. The Higgs boson was allowed to decay to $\tau^{+} \tau^{-}$or $W^{+} W^{-}$with subsequent decay of the $W$ bosons to $e \nu_{e}, \mu \nu_{\mu}$ and $\tau \nu_{\tau}$.

In all cases of signal and background the full parton shower, hadronization and the underlying event [53] were included. ${ }^{3}$ All the runs have been generated using the MSTW2008nlo 68\% PDF set. We note that we do not consider pure QCD-initiated backgrounds since these are expected to be negligible in the high-missing transverse momentum regime, particularly in conjunction with the existence of isolated leptons or $\tau \mathrm{s}$.

We define a SUSY benchmark point C350-100, with parameters

$$
M_{2}=m_{\tilde{\chi}_{1}^{ \pm}}=m_{\tilde{\chi}_{2}^{0}}=350 \mathrm{GeV}, \quad M_{1}=m_{\tilde{\chi}_{1}^{0}}=100 \mathrm{GeV}
$$

This point will be used as an example to demonstrate the effect of cuts and provide a typical point to aid the development of the strategy for discriminating the signal against the various backgrounds.

\footnotetext{
${ }^{3}$ The event generator dependence is beyond the scope of this phenomenological study. Moreover, we note that do not include a description of pile-up events. These should be considered in detail in a full experimental simulation.
} 


\subsection{Tau identification}

\subsubsection{Tau lepton decay modes}

The study of final states containing hadronically decaying $\tau$ leptons is an important and growing part of the LHC's physics program. The $\tau$ lepton has a multitude of decay modes, which we may split these into two categories: 'leptonic', if the visible decay products contain a single lepton, and 'hadronic', if there are one or three charged hadrons present. We label the corresponding modes $\tau_{\ell}$ and $\tau_{h}$ respectively. The hadronic modes are also categorised as '1-prong' and '3-prong', according to the number of charged particles involved in the decay. ${ }^{4}$ The label ' $\ell$ ' here and elsewhere implies an electron or a muon. The branching ratios for these modes are: ${ }^{5}$

- leptonic: $\operatorname{BR}\left(\tau \rightarrow \tau_{\ell}\right) \sim 0.35$.

- hadronic: $\operatorname{BR}\left(\tau \rightarrow \tau_{h}\right) \sim 0.625$.

These imply, for a Higgs boson decay to $\tau^{+} \tau^{-}$:

- $\operatorname{BR}\left(h \rightarrow \tau_{h} \tau_{h}\right) \sim 0.39 \times B R\left(h \rightarrow \tau^{+} \tau^{-}\right)$.

- $\operatorname{BR}\left(h \rightarrow \tau_{h} \tau_{\ell}\right) \sim 0.44 \times B R\left(h \rightarrow \tau^{+} \tau^{-}\right)$.

- $\operatorname{BR}\left(h \rightarrow \tau_{\ell} \tau_{\ell}\right) \sim 0.12 \times B R\left(h \rightarrow \tau^{+} \tau^{-}\right)$.

\subsubsection{Hadronic tau identification algorithm}

Both ATLAS [55] and CMS [56] employ reconstruction and identification algorithms, used to identify hadronically decaying $\tau$ leptons and reject various backgrounds. Here, we do not attempt to reproduce either of the ATLAS or CMS algorithms exactly, but instead use elements from both resulting in an algorithm that we expect performs in an equivalent way. We also borrow elements from [57], which examines di- $\tau$ tagging in the boosted regime. ${ }^{6}$ The resulting algorithm is expected to provide conservative hadronic $\tau$-tagging results, and could be improved substantially via the use of boosted decision trees (BDT) or other advanced multivariate methods. Since we will not employ simulation of detector effects in the present analysis, we focus on a simple cut-based algorithm for simplicity.

The first part of the basic algorithm for hadronic $\tau$ identification proceeds as follows:

- Reconstruct jets with $R=0.5$ using the Cambridge/Aachen jet algorithm as implemented in FastJet [59]. An individual jet is then investigated for constituent hadronic tracks. ${ }^{7}$

\footnotetext{
${ }^{4}$ If one of the taus undergoes a 3-prong decay, one may improve the analysis significantly using the information of the secondary vertex of the 3-prong tau decay [54]. This requires a dedicated study and we do not use the secondary vertex information in this paper.

${ }^{5}$ These do not add up to $100 \%$, since we are only considering the dominant 1-prong and 3-prong decay modes.

${ }^{6}$ For di- $\tau$ tagging in Higgs searches, see also ref. [58].

${ }^{7}$ Usage of the word "track" here and elsewhere in this article implies "charged particle".
} 
- Consider a track to be a 'seed' if it is the hardest track in the jet, has $p_{T}>5 \mathrm{GeV}$ and is within $\Delta R=0.1$ of the jet axis.

- If such a track is found, one defines inner and outer cones around it. We use $R_{\text {in }}=0.2$ and $R_{\text {out }}=0.4$ respectively.

- Require no photons with $p_{T}>2 \mathrm{GeV}$ and no charged tracks with $p_{T}>1 \mathrm{GeV}$ to lie within the defined annulus between $R_{\text {in }}$ and $R_{\text {out }}$.

The basic part of the algorithm itself does not provide satisfactory rejection against the QCD jet background to hadronically decaying $\tau$ leptons. If a jet satisfies all the above criteria, then the following variables are constructed:

- $\Delta R_{\max }$ : the distance to the track furthest away from the jet axis.

- $f_{\text {core }}$ : the fraction of the total jet energy contained in the centre-most cone defined by $\Delta R<0.1$.

These variables provide strong discriminating power against QCD jets $[55,60] .{ }^{8}$ To perform the rejection of QCD jets, here we apply the following cuts:

- $\Delta R_{\max }<0.05$.

- $f_{\text {core }}>0.95$.

In figure 6 we show the variables $\Delta R_{\max }$ and $f_{\text {core }}$, constructed for hadronic jets for a signal benchmark point $\mathbf{C 3 5 0 - 1 0 0}$ and the $W+$ jets background. ${ }^{9}$ Only jets with $p_{T}>$ $20 \mathrm{GeV}$ and $|\eta|<2.5$ were considered. In figure 7 we show the efficiency of $\tau$ identification versus the transverse momentum of the jet in question, $p_{T, \text { jet }}$, obtained by the procedure outlined in this section. For the signal, the efficiency was defined for the identification of 'true' $\tau$ jets, defined to be those closest to the visible $\tau$ decay products taken from the Monte Carlo truth. For the $W+$ jets background, the efficiency was defined with respect to any jet. The efficiency for the SUSY benchmark point C350-100 varies from around 50\% in the $p_{T \text {,jet }}$ region of $20-300 \mathrm{GeV}$ and then drops down to $\sim 20 \%$ at around $p_{T \text {,jet }} \sim 400 \mathrm{GeV}$. For the $W+$ jets background the efficiency starts off at $\sim 1 \%$ at $p_{T, \text { jet }} \sim 20 \mathrm{GeV}$ and then rises to an efficiency of $2-3 \%$, more or less constant up to $p_{T \text {,jet }} \sim 500 \mathrm{GeV}$.

\subsection{Analysis}

In order not to limit the scope of our phenomenological analysis, we will assume that a multi-object trigger can conceivably be designed, involving multiple leptons or hadronic tau jets with $p_{T} \gtrsim 20 \mathrm{GeV}$, in conjunction with missing transvese energy $\mathbb{E}_{T} \gtrsim 100 \mathrm{GeV}[61-$ 63]. Such a trigger actually appears in the ATLAS study of ref. [64], where in section 3.4 , pre-selection critera of $p_{T}$ (lep) $>10 \mathrm{GeV}, p_{T}($ tau $)>20 \mathrm{GeV}$ and $\mathbb{E}_{T}>100 \mathrm{GeV}$

\footnotetext{
${ }^{8}$ More variables have been employed by the experimental collaborations, but we found that the two that we consider are sufficient at this level of simulation.

${ }^{9}$ Two-dimensional distributions of the two variables for the two samples that we have investigated can be seen in appendix A.
} 

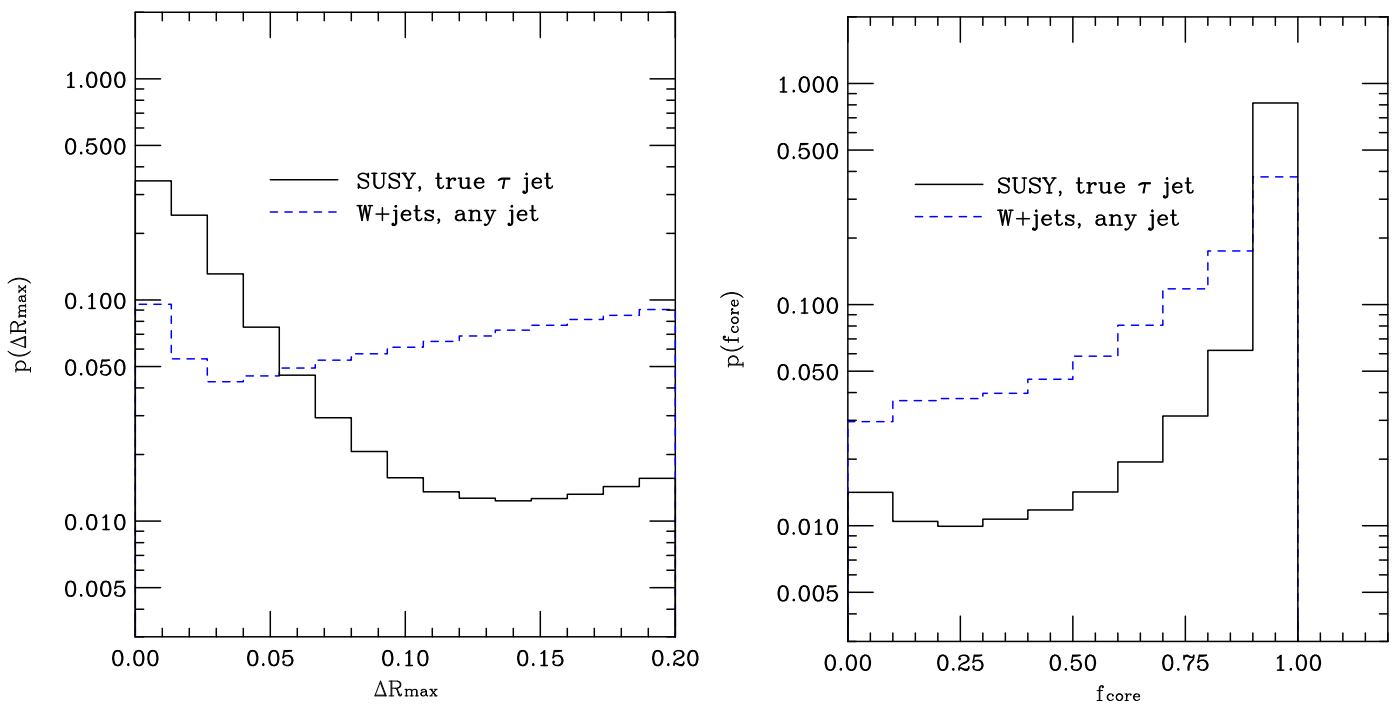

Figure 6. Distributions of the variables used for discrimination of the jets originating from $\tau$ leptons and those from QCD, for the SUSY benchmark point C350-100 and the $W+$ jets background $\left(W \rightarrow e \nu_{e} / \mu \nu_{\mu}\right)$.

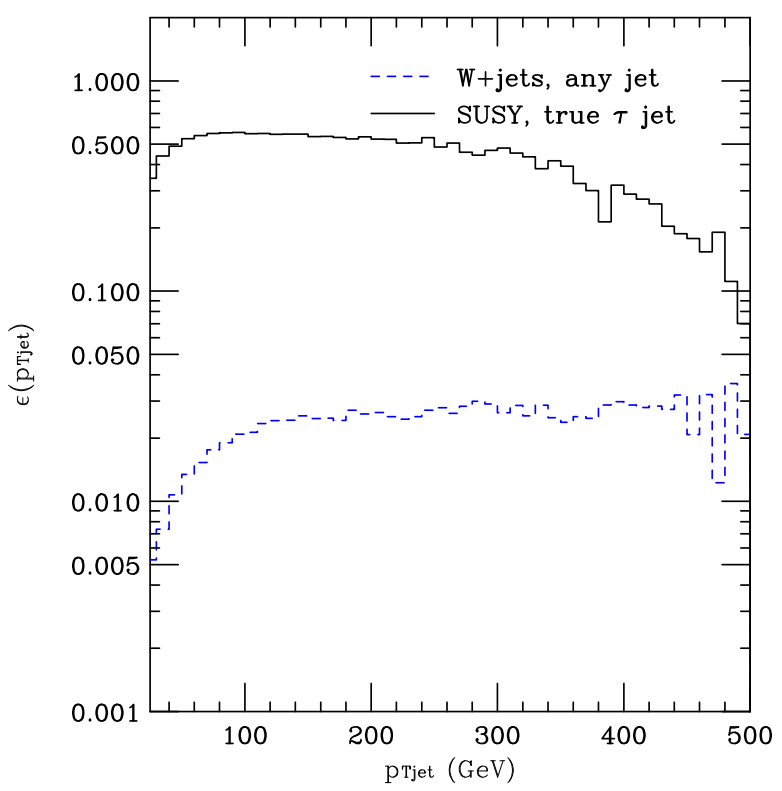

Figure 7. The efficiency of tagging a jet as a $\tau$-jet for the SUSY benchmark point C350-100 and the $W+$ jets background (with $W \rightarrow e \nu_{e} / \mu \nu_{\mu}$ ). For C350-100, the efficiency was defined for the identification of 'true' $\tau$ jets, defined to be those closest to the visible $\tau$ decay products taken from the Monte Carlo truth. For the $W+$ jets, the efficiency was defined with respect to any jet. Jets of $p_{T}>20 \mathrm{GeV}$ and $|\eta|<2.5$ are considered in both cases. 
are imposed for a similar Wh-mediated SUSY search at the high-luminosity LHC. Our selection criteria are essentially tighter than their pre-selection criteria. We investigate the effect of increasing the $p_{T}$ thresholds for leptons and taus below.

We define the first level of the analysis for discriminating the signal against the various backgrounds as follows:

1. Particles of $p_{T}>0.5 \mathrm{GeV}$ and $|\eta|<5.0$ are considered.

2. If isolated leptons with $p_{T}>20 \mathrm{GeV}$ are found, they are placed in a separate list, and removed from the list of particles. An isolated lepton is defined as either: having $\sum_{i} p_{T, i}$ less than $20 \%$ of its transverse momentum around a cone of $\Delta R=0.4$ around it, or as a lepton that contains no photons with $p_{T}>2 \mathrm{GeV}$ and no tracks with $p_{T}>1 \mathrm{GeV}$ in the annulus $\Delta R=(0.2,0.4)$ around it. ${ }^{10}$

3. Jet finding is performed on the list of remaining particles, using Fast Jet and the Cambridge/Aachen jet algorithm, with parameter $R=0.5$. Jets of $p_{T}>20 \mathrm{GeV}$ are accepted.

4. Tagging of $\tau$-jets is performed as described in section 3.2 .

5. Only events with a total number of isolated leptons, $n_{\ell, \text { iso }}$, and $\tau$-tagged jets, $n_{\tau, \text { tag }}$, equal to 3 are accepted: i.e. we require $n_{\tau, \text { tag }}+n_{\ell \text {,iso }}=3$. A hypothesis is then performed to match the topology of the SUSY events. The hypotheses vary according to the number of isolated leptons and $\tau$-tagged jets and are listed in detail in Table 2.

6. Several variables are calculated and are passed through to the second level of analysis.

Steps 1-5 are what we define as the 'basic' analysis, and these will provide what we call $\sigma_{\text {basic }}($ table 4 ). The variables calculated in step 6 and used for further discrimination in the second-level analysis are: the transverse momentum of the di- $\tau$-tagged system, $p_{T, \tau \tau}$, the distance between the $\tau$-tagged jets, $\Delta R_{\tau, \tau}$, the distance between the di- $\tau$-tagged system and the lepton, $\Delta R_{\tau \tau, \ell}$, the missing transverse energy, $\not p_{T}$ and the variable $M_{\min }$, which is sharply peaked at low values for the $W Z$ background and broadly falls off for the signal, defined in appendix B. The variables are outlined in table 3 . There we provide an example set of cuts, applied to the SUSY benchmark point C350-100, found to give a significance of $\sim 2.5 \sigma$ for an integrated luminosity of $100 \mathrm{fb}^{-1}$ at $14 \mathrm{TeV}$. To investigate the effect of higher $p_{T}$ thresholds, we set for each isolated lepton, $p_{T}$ (lept) $>30 \mathrm{GeV}$, and for each hadronic tau, $p_{T}(\mathrm{tau})>40 \mathrm{GeV}$. This reduces the significance to $\sim 2 \sigma$ and verifies that our analysis is not substantially affected by higher thresholds that aim to take into account current triggering strategies.

For completeness, we show in table 4 the resulting cross sections after applying the analysis on the SUSY benchmark point and the different backgrounds for this example. In the final stage of the analysis the $n_{\tau, \text { tag }}=3$ channel was excluded, since it was found to reduce significance by allowing more background. Note that this set of cuts will constitute 'signal region 1' of our full analysis.

\footnotetext{
${ }^{10}$ We apply two different criteria to take into account the possibility of radiation from the core lepton.
} 


\begin{tabular}{|c|c|c|c|}
\hline$n_{\tau, \operatorname{tag}}$ & $n_{\ell, \text { iso }}$ & real signal channels & hypothesis \\
\hline 3 & 0 & $\left(h \rightarrow \tau_{h} \tau_{h}, W \rightarrow \tau \nu\right)$ & assign hardest two to $h$. \\
\hline 2 & 1 & $\begin{array}{l}\left(h \rightarrow \tau_{h} \tau_{\ell}, W \rightarrow \tau_{h} \nu_{\tau}\right) \\
\left(h \rightarrow \tau_{h} \tau_{h}, W \rightarrow \ell \nu_{\ell}\right)\end{array}$ & assign hardest two to $h$. \\
\hline 1 & 2 & $\begin{array}{l}\left(h \rightarrow \tau_{h} \tau_{\ell}, W \rightarrow \ell \nu_{\ell}\right) \\
\left(h \rightarrow \tau_{\ell} \tau_{\ell}, W \rightarrow \tau_{h} \nu_{\tau}\right)\end{array}$ & $\begin{array}{l}\text { if leptons are same sign, assign } \\
\text { highest- } p_{T} \text { to } h \text { along with the } \\
\tau \text {-tagged jet. Otherwise: as- } \\
\text { sign any two highest- } p_{T} \text { to } h \text {. }\end{array}$ \\
\hline 0 & 3 & $\left(h \rightarrow \tau_{\ell} \tau_{\ell}, W \rightarrow \ell \nu_{\ell}\right)$ & $\begin{array}{l}\text { If all leptons are the same sign, } \\
\text { reject the event. Otherwise: } \\
\text { pair two highest- } p_{T} \text { of opposite } \\
\text { sign as the } h .\end{array}$ \\
\hline
\end{tabular}

Table 2. The hypotheses applied for the reconstruction of the Supersymmetric topology as described in the main text. The different hypotheses are given according to the number of $\tau$-tagged jets, $n_{\tau, \text { tag }}$, and the number of isolated leptons $n_{\ell, \text { iso }}$. In the final stage of the analysis, the $n_{\tau, \text { tag }}=3$ was found to reduce significance and was not considered.

Details of how the initial cross sections for the signal and background are calculated are given in appendix C. We note that in the case of the $Z+$ jets and $W+$ jets samples, we obtained $N_{\text {cuts }}=0$ events after all cuts. ${ }^{11}$ To provide an estimate of the cross section, we assume that the Poisson distribution has mean number of events $\lambda=3$ and use this as an upper bound to estimate the resulting cross sections. The probability of having a Poisson-distributed sample with mean $\lambda>3$, given that zero events have been observed, is $\simeq 0.05$. It is useful to mention at this point that we do not apply a $K$-factor to the $Z+$ jets or $W+$ jets cross sections. The induced uncertainty due to this omission can be absorbed in the systematic uncertainty due to lack or low number of events in the final Monte Carlo samples. Nevertheless, since conservative estimates for these backgrounds have been assumed, $K$-factors of $\sim 2$ would not have a significant impact to the main conclusions of our analysis.

\subsection{Signal regions}

To perform a scan of the supersymmetric parameter space, we define signal regions, with cuts that aim to bring out the different qualities of the defined variables. These signal regions are shown in table 5 , for the variables defined in table 3. All the signal regions exclude the $n_{\tau, \text { tag }}=3$ channel, since it was found to reduce significance.

\section{Results}

We performed the analysis on the $M_{2}-M_{1}$ plane, according to the cuts defined in the signal regions in table 5 at integrated luminosities of $100 \mathrm{fb}^{-1}, 300 \mathrm{fb}^{-1}$ and $3000 \mathrm{fb}^{-1}$. We show

\footnotetext{
${ }^{11}$ For the $Z\left(\rightarrow \tau^{+} \tau^{-}\right)+$jets, this depends on how large the missing transverse momentum cut imposed is.
} 


\begin{tabular}{|lll|}
\hline variable & definition & benchmark point cut (三signal region 1$)$ \\
\hline$\not p_{T}$ & missing transverse momentum & $>95 \mathrm{GeV}$ \\
$M_{\text {min }}$ & appendix B & $>235 \mathrm{GeV}$ \\
$p_{T, \tau \tau}$ & di- $\tau$-tagged jet $p_{T}$ & $>20 \mathrm{GeV}$ \\
$\Delta R_{\tau, \tau}$ & distance between $\tau$-tagged jets & $\in(0.1,2.9)$ \\
$\Delta R_{\tau \tau, \ell}$ & distance between di- $\tau$-tagged jet system & $\in(0.1,2.6)$ \\
& and lepton & \\
\hline
\end{tabular}

Table 3. The variables used for further discrimination after the basic part of the analysis is applied to the signal and backgrounds.

\begin{tabular}{|c|c|c|c|}
\hline sample & $\sigma_{\text {initial }}(\mathrm{fb})$ & $\sigma_{\text {basic }}(\mathrm{fb})$ & $\sigma_{\text {cuts }}(\mathrm{fb})$ \\
\hline $\begin{array}{l}\text { SUSY C350- } \\
100\end{array}$ & 5.7 & 0.658 & 0.152 \\
\hline$W Z$ & 767 & 85.734 & 0.079 \\
\hline$W\left(\rightarrow \ell \nu_{\ell}\right)+$ jets & $\sim 600 \times 10^{3}$ & 61.974 & $\lesssim 0.055$ \\
\hline $\begin{array}{l}W(\rightarrow \\
\left.\tau \nu_{\tau}\right)+ \text { jets }\end{array}$ & $\sim 300 \times 10^{3}$ & 7.591 & $\lesssim 0.052$ \\
\hline$h V$ & 443 & 5.071 & 0.037 \\
\hline$t \bar{t} h$ & 3.4 & 0.147 & 0.008 \\
\hline$t \bar{t}$ & 8600 & 14.876 & 0.005 \\
\hline$Z(\rightarrow \ell \ell)+$ jets & $\sim 600 \times 10^{3}$ & 1659 & $\lesssim 0.029$ \\
\hline$Z(\rightarrow \tau \tau)+$ jets & $\sim 300 \times 10^{3}$ & 52.762 & 0.047 \\
\hline
\end{tabular}

Table 4. The effect of the cuts on the SUSY benchmark point C350-100 and the relevant backgrounds. The 'initial' cross section calculations are presented in appendix C. The 'basic' cross sections are obtained after applying the procedure outlined in steps 1-5 in section 3.3.

\begin{tabular}{|llllllll|}
\hline variable & SR1 & SR2 & SR3 & SR4 & SR5 & SR6 & SR7 \\
\hline$\not p_{T}$ & $95 \mathrm{GeV}$ & $120 \mathrm{GeV}$ & $100 \mathrm{GeV}$ & $90 \mathrm{GeV}$ & $90 \mathrm{GeV}$ & $150 \mathrm{GeV}$ & $90 \mathrm{GeV}$ \\
$M_{\min }$ & $235 \mathrm{GeV}$ & $270 \mathrm{GeV}$ & $220 \mathrm{GeV}$ & $220 \mathrm{GeV}$ & $300 \mathrm{GeV}$ & $240 \mathrm{GeV}$ & $200 \mathrm{GeV}$ \\
$p_{T, \tau \tau}$ & $20 \mathrm{GeV}$ & $80 \mathrm{GeV}$ & $20 \mathrm{GeV}$ & $50 \mathrm{GeV}$ & $20 \mathrm{GeV}$ & $20 \mathrm{GeV}$ & $20 \mathrm{GeV}$ \\
$\Delta R_{\tau, \tau}$ & $(0.1,2.9)$ & $(0.1,2.9)$ & $(0.1,2.9)$ & $(0.1,2.9)$ & $(0.1,2.9)$ & $(0.1,2.9)$ & $(0.1,2.9)$ \\
$\Delta R_{\tau \tau, \ell}$ & $(0.1,2.6)$ & $(0.1,2.5)$ & $(0.1,2.6)$ & $(0.1,2.6)$ & $(0.1,2.6)$ & $(0.1,2.6)$ & $(0.1,2.6)$ \\
\hline
\end{tabular}

Table 5. The cuts for the different signal regions (SR) used in the analysis. 
the resulting envelope of significances in figure 8 , where the solid curves show the $3 \sigma$ evidence region, whereas the dashed curves show the $5 \sigma$ discovery region. We also show in figure 9, the expected exclusion region at $2 \sigma$ (solid) and $3 \sigma$ (dashed). For completeness, we show the corresponding overlapping signal regions in appendix F. There, we also provide the total cross sections for the backgrounds after cuts given by the different signal regions. These can be used to infer constraints in explicit SUSY models that contain the specific decay chain we are considering.

The analysis can yield a low number of events for both signal and background, of $\mathcal{O}(10)$, and for the calculation of significance we used the Poisson distribution to calculate the $p$-values. These were subsequently converted to the corresponding Gaussian standard deviations. Details of the procedure are provided in appendix D, with supplementary material in appendix E.

Although the authors of ref. [34] have not performed an equivalent parameter-space scan over $M_{1}-M_{2}$, and the details of the chosen parameters differ from the ones presented in this article, it is still interesting to compare with the potential of the final state in which the channel $\tilde{\chi}_{2}^{0} \tilde{\chi}_{1}^{ \pm} \rightarrow\left(h \tilde{\chi}_{1}^{0}\right)\left(W^{ \pm} \tilde{\chi}_{1}^{0}\right)$ involves leptonic $W$ decays and Higgs boson decays to $b \bar{b}$. There, the authors have found that it is possible to discover a signal of the process at the $\sim 5 \sigma$ level at $\sim 100 \mathrm{fb}^{-1}$ of luminosity, for points for which $M_{2} \sim 265-390 \mathrm{GeV}$ and $M_{1} \sim 133-198 \mathrm{GeV}$. Indeed, our analysis is competitive with this result, with such points falling somewhere between the $3 \sigma$ and $5 \sigma$ discovery regions at $100 \mathrm{fb}^{-1}$ and $300 \mathrm{fb}^{-1}$, as demonstrated by the black and red curves respectively, in figure 8 . This indicates that this channel is as important as the final state with $h \rightarrow b \bar{b}$, or at least complementary.

\section{Conclusions}

We have presented a phenomenological analysis of the channel $\tilde{\chi}_{2}^{0} \tilde{\chi}_{1}^{ \pm} \rightarrow\left(h \tilde{\chi}_{1}^{0}\right)\left(W^{ \pm} \tilde{\chi}_{1}^{0}\right)$ using the $W \rightarrow \ell \nu_{\ell} / \tau \nu_{\tau}$ and Higgs boson channels $\left(h \rightarrow \tau^{+} \tau^{-}\right.$and $h \rightarrow W^{+} W^{-} \rightarrow$ leptons $)$ at the LHC. Such channels are common in many concrete SUSY models where the predictions include $\tilde{\chi}_{2}^{0}$ and $\tilde{\chi}_{1}^{ \pm}$that predominantly decay into $h$ and $W$, respectively.

Our analysis has included detailed hadron-level simulation of the relevant dominant backgrounds, including the effects of the underlying event. Hadronic $\tau$ identification was modelled at hadron level with a custom-made algorithm based on the ones employed by both the ATLAS and CMS experiments. We have employed a cut-based analysis on several variables that bring out the properties of the signal against those of the backgrounds. Specifically, we have constructed a mass variable, $M_{\min }$, which is sharply peaked at low value for the $W Z$ background and broadly falls off for the signal.

Consequently we have demonstrated the potential for discovering or constraining the SUSY parameter space in the $M_{2}-M_{1}$ plane at integrated luminosities of $100 \mathrm{fb}^{-1}, 300 \mathrm{fb}^{-1}$ and $3000 \mathrm{fb}^{-1}$, collected at a $14 \mathrm{TeV}$ proton-proton centre-of-mass energy. The $5 \sigma$ discovery potential of our analysis reaches up to $M_{2} \simeq 350 \mathrm{GeV}$ with $M_{1} \lesssim 100 \mathrm{GeV}$ at the $14 \mathrm{TeV}$ LHC with $300 \mathrm{fb}^{-1}$. This implies that a future $e^{+} e^{-}$collider with $\sqrt{s}=1 \mathrm{TeV}$ can play indispensable role to cover $M_{2}<500 \mathrm{GeV}$ region. A large part of this region can also 


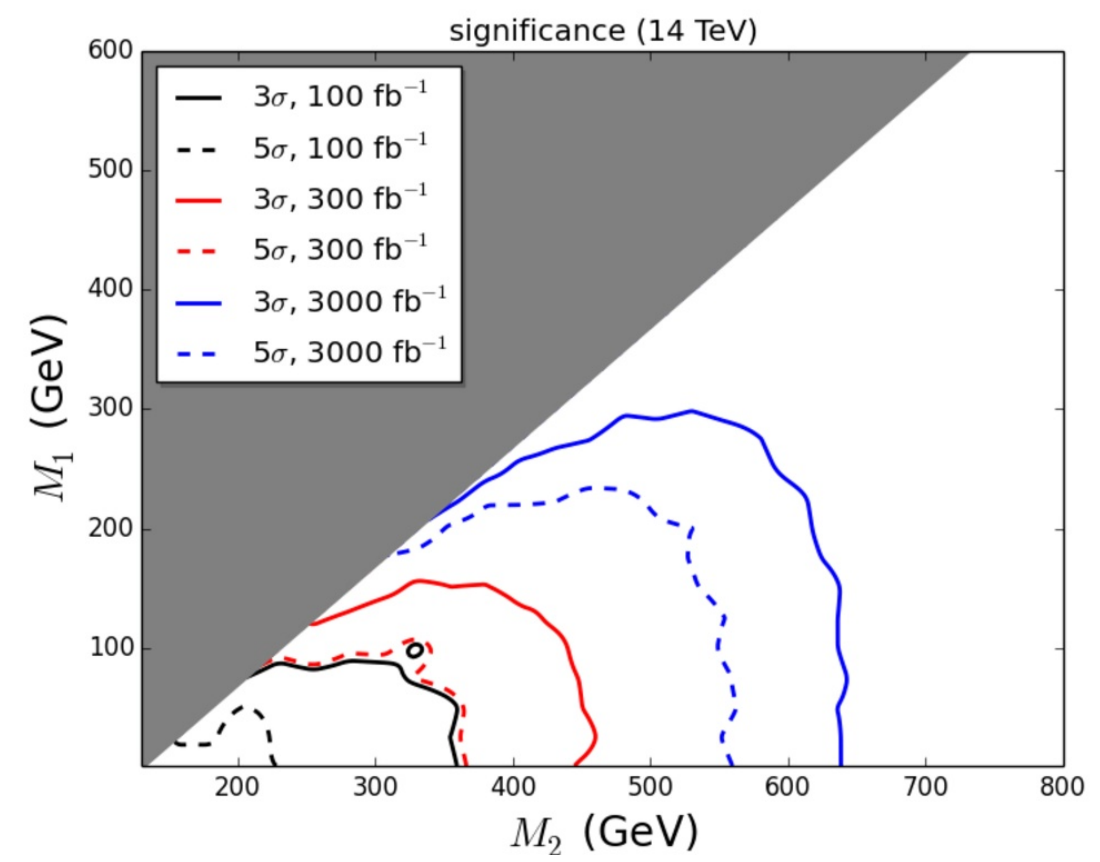

Figure 8. The significance envelope on the $M_{2}-M_{1}$ plane obtained for the signal regions defined in table 5 at integrated luminosities of $100 \mathrm{fb}^{-1}$ (black) or $300 \mathrm{fb}^{-1}$ (red). The solid curves show the $3 \sigma$ evidence region, whereas the dashed curves show the $5 \sigma$ discovery region.

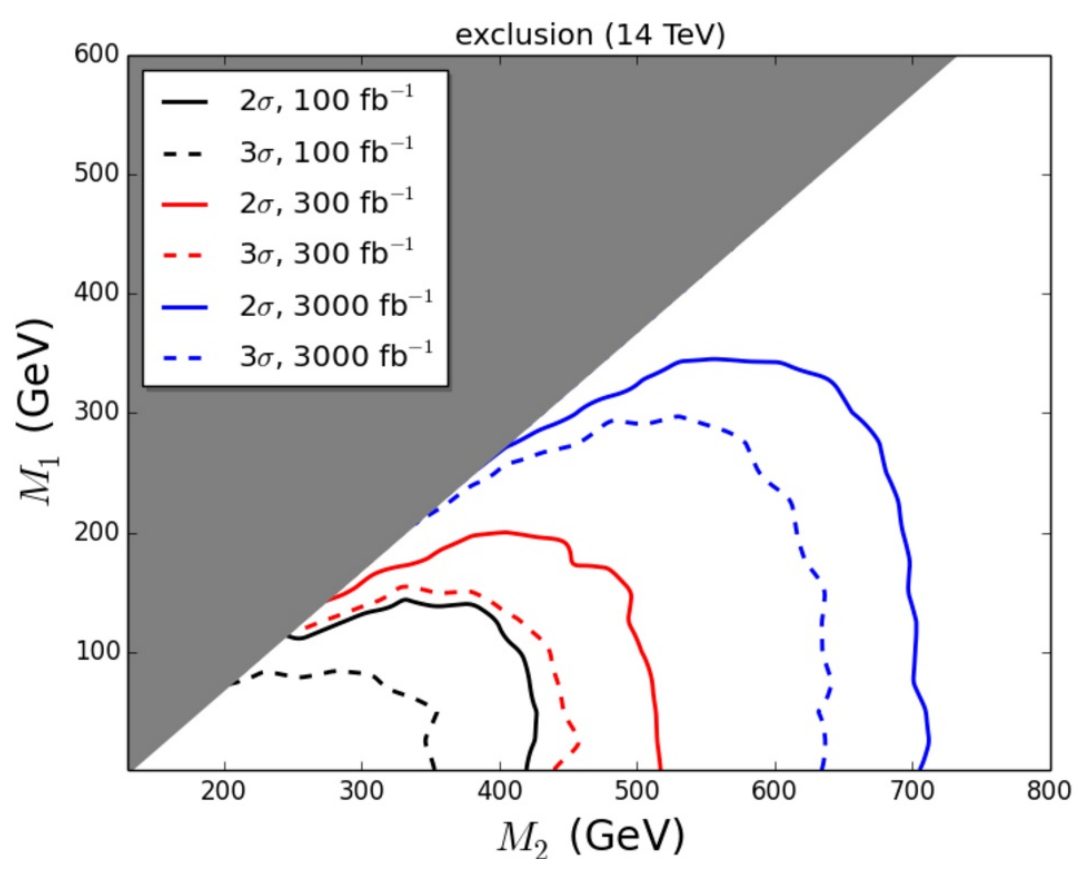

Figure 9. The exclusion envelope on the $M_{2}-M_{1}$ plane obtained for the signal regions defined in table 5 at integrated luminosities of $100 \mathrm{fb}^{-1}$ (black) or $300 \mathrm{fb}^{-1}$ (red). The solid curves show the $2 \sigma$ exclusion boundary, whereas the dashed curves show the $3 \sigma$ boundary. 
be covered by the $14 \mathrm{TeV}$ High Luminosity LHC with $3000 \mathrm{fb}^{-1}$, which has a discovery potential in the $M_{2} \lesssim 550 \mathrm{GeV}, M_{1} \lesssim 200 \mathrm{GeV}$ region.

This work serves a first study of making use of $h \rightarrow \tau \tau$ mode in the chargino-neutralino searches in future runs of the LHC. We thus recommend further examination of this channel by experimental collaborations, including the effects of full detector simulation, $\tau$-jet tagging and multi-variate analyses.

\section{A Correlation between $\tau$-tagging variables}

We show in figure 10 the two-dimensional distributions of the two variables, $\Delta R_{\max }$ and $f_{\text {core }}$, that we have employed for the discrimination of hadronic tau jets from QCD jets. The variables are correlated in both signal and background. However, it is evident that both variables are necessary for discrimination between the hadronic taus and the QCD jets. The figure has been normalised to integrate to unity over the range $\left(\Delta R_{\max }, f_{\text {core }}\right)=(0.2,1.0)$ and hence one can notice that the SUSY signal is more sharply peaked in the region $\left(\Delta R_{\max }<0.5, f_{\text {core }}>0.95\right)$.

\section{B Definition of the $M_{\min }$ variable}

We define the $M_{\min }$ variable that we will use as a handle for rejecting non-SUSY backgrounds. Although the variable is designed to reject the $W Z$ background, it can also potentially perform well against other backgrounds. There are three neutrinos in the final state: one coming from $W$ decay, the other two from the $\tau$ lepton decays. The direction of the $\tau$-neutrino is approximately collimated with respect to the original $\tau$ lepton direction due to the mass hierarchy, $m_{Z} \gg m_{\tau}$. With this approximation, the momenta of the $\tau$ lepton and the $\tau$-neutrino can be parametrised as

$$
\begin{aligned}
p_{\tau^{+}} & =p_{\rho_{1}} / a, & p_{\tau^{-}} & =p_{\rho_{2}} / b, \\
p_{\nu_{1}} & =(1 / a-1) p_{\rho_{1}}, & p_{\nu_{2}} & =(1 / b-1) p_{\rho_{2}},
\end{aligned}
$$

where $p_{\rho_{1 / 2}}$ is the momentum of the visible decay products and: $0<a(b)<1$. Note that events that in the phenomenological analysis of this article that do not satisfy this condition on $a$ and $b$ are deemed 'unphysical' and rejected. Assuming the event topology in figure 11 , the unknown neutrino momenta can be constrained by the mass shell conditions of the $W$ and $Z$ bosons and the missing momentum conditions. ${ }^{12}$

$$
\begin{array}{r}
a, b, \mathbf{p}_{\nu}: 5 \text { unknowns } \\
m_{Z}, m_{W}, p_{\text {miss }}^{x}, p_{\text {miss }}^{y}: 4 \text { constraints }
\end{array}
$$

Since (\# of unknown - \# of constraints) $=1$, we can parameterise the all neutrino momenta by a single parameter, $\theta$.

\footnotetext{
${ }^{12}$ Vectors in bold typeset represent 3 -vectors.
} 

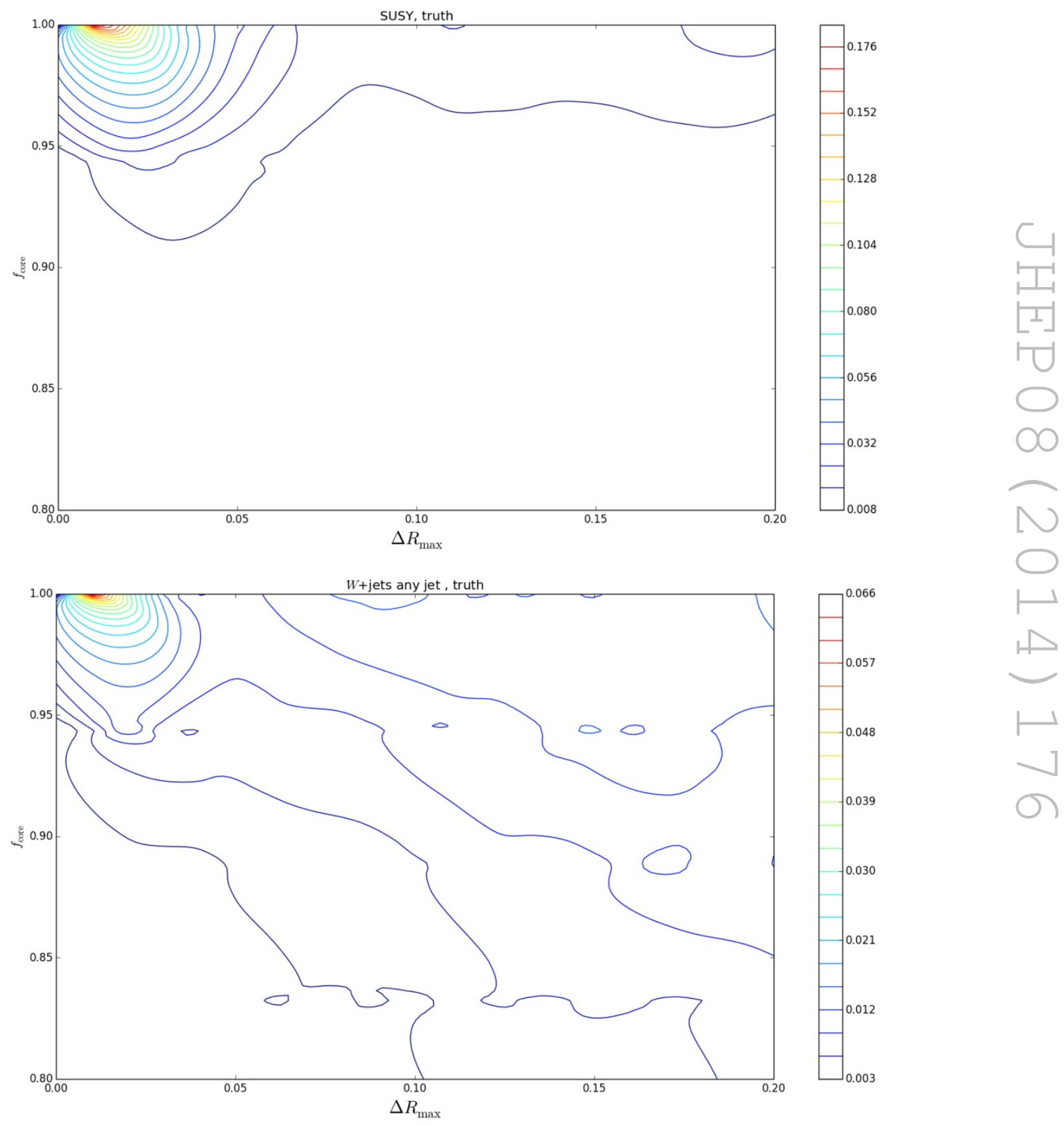

Figure 10. Two dimensional-distributions of the variables used for discrimination of the jets originating from $\tau$ leptons and those from QCD, $\Delta R_{\max }$ and $f_{\text {core. }}$ top: SUSY benchmark point C350-100, bottom: $W+$ jets background $\left(W \rightarrow e \nu_{e} / \mu \nu_{\mu}\right)$. 


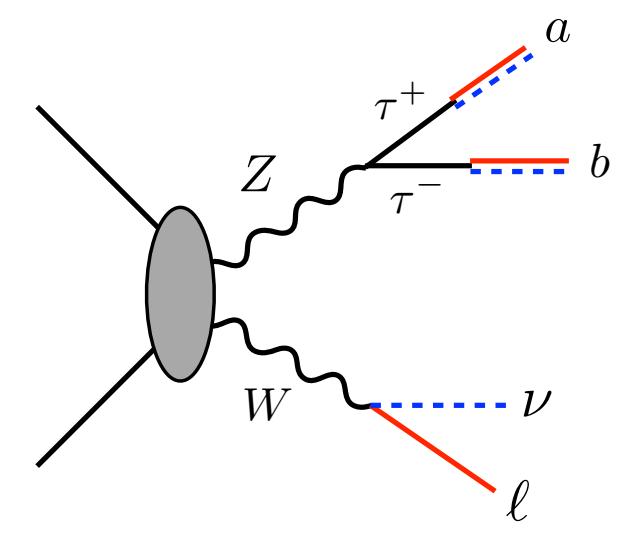

Figure 11. The $W Z$ background topology considered in constructing the $M_{\min }$ variable.

The mass-shell constraint for the $Z$ boson gives

$$
a b=\frac{2\left(p_{\rho_{1}} \cdot p_{\rho_{2}}\right)}{m_{Z}^{2}} .
$$

By introducing $\theta \equiv \arctan \left(\frac{a}{b}\right), a$ and $b$ can be written as

$$
a=\sqrt{\frac{2\left(p_{\rho_{1}} \cdot p_{\rho_{2}}\right)}{m_{Z}^{2}} \tan \theta}, \quad b=\sqrt{\frac{2\left(p_{\rho_{1}} \cdot p_{\rho_{2}}\right)}{m_{Z}^{2}} \tan ^{-1} \theta}
$$

The transverse components of the neutrino momentum are determined by

$$
\mathbf{p}_{\nu}^{\mathbf{T}}=\mathbf{p}_{\text {miss }}^{\mathbf{T}}-(1 / a-1) \mathbf{p}_{\rho_{1}}-(1 / b-1) \mathbf{p}_{\rho_{2}} .
$$

The mass shell condition of $W$ constrains the last unknown parameter $p_{\nu}^{z}$ as

$$
p_{\nu}^{z \pm}=\frac{c p_{\ell}^{z} \pm \sqrt{E_{\ell}^{2}\left(c^{2}-t_{\ell}^{2} t_{\nu}^{2}\right)}}{t_{\ell}^{2}},
$$

where $\mathbf{t}_{\ell / \nu}=\mathbf{p}_{\ell / \nu}^{T}, c=\mathbf{t}_{\ell} \cdot \mathbf{t}_{\nu}+m_{W}^{2} / 2$. If eq. (B.5) yields complex solution, we simply take the real part $[65,66]$.

All the neutrino momenta are now parametrised by $\theta$. We define the invariant mass of the system

$$
M_{\mathrm{inv}}^{ \pm}(\theta)=\sqrt{\left[p_{\ell}+p_{\nu}^{ \pm}(\theta)+p_{\tau^{+}}(\theta)+p_{\tau^{-}}(\theta)\right]^{2}}
$$

where \pm corresponds to the discrete ambiguity in eq. (B.5). The variable $M_{\min }$ is defined by the global minimum of the $M_{\text {inv }}$ over the $\theta$

$$
M_{\mathrm{min}} \equiv \min _{\theta \in[0, \pi / 2]} \min \left\{M_{\mathrm{inv}}^{+}(\theta), M_{\mathrm{inv}}^{-}(\theta)\right\} .
$$

Figure 12 shows the distributions of $M_{\min }$ for the $W Z$ and SUSY benchmark point event samples for 1000 parton-level events. The SUSY benchmark point C350-100 involves the parameters:

$$
m_{\tilde{\chi}_{1}^{ \pm}}=m_{\tilde{\chi}_{2}^{0}}=350 \mathrm{GeV}, \quad m_{\tilde{\chi}_{1}^{0}}=100 \mathrm{GeV} .
$$




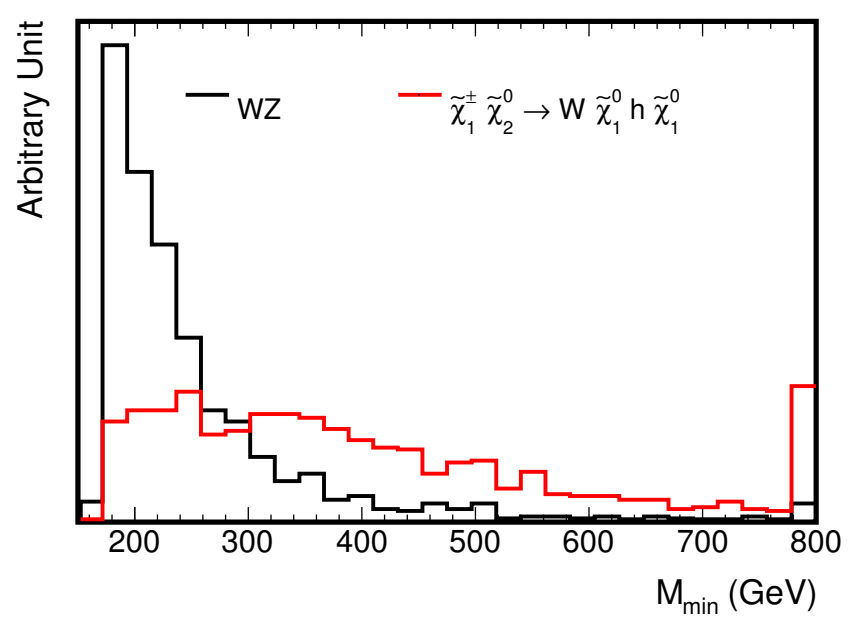

Figure 12. The $M_{\min }$ distribution for $W Z$ (black) and SUSY benchmark point (red) event samples.

\section{Calculation of the initial cross sections}

For completeness we provide the branching ratios used to reproduce the initial cross sections that appear in table 4 .

- SUSY benchmark C350-100: using Prospino 2.1, the NLO cross section for the SUSY benchmark point is $\sigma_{S U S Y} \simeq 200 \mathrm{fb}$. For the signal, we consider the decays of the $W$ to all three lepton families and the decays of the Higgs boson to either $\tau^{+} \tau^{-}$or $W^{+} W^{-}$(again with the $W$ s decaying to all leptons). We also assume that $B R\left(\tilde{\chi}_{2}^{0} \rightarrow h \tilde{\chi}_{1}^{0}\right)=1$. Hence:

$$
\begin{aligned}
\sigma(S U S Y)_{\text {initial }}= & \sigma_{S U S Y} \times B R(W \rightarrow \ell / \tau \nu) \\
& \times\left(B R\left(h \rightarrow \tau^{+} \tau^{-}\right)+B R\left(h \rightarrow W^{+} W^{-}\right) \times B R(W \rightarrow \ell / \tau \nu)^{2}\right) \\
= & \sigma_{S U S Y} \times 0.3257 \\
& \times\left(0.0632+0.2155 \times 0.3257^{2}\right) \\
\simeq & 5.7 \mathrm{fb}
\end{aligned}
$$

- $W Z$ : we allow for $\left(W \rightarrow \ell \nu, Z \rightarrow \tau^{+} \tau^{-}\right)$or $\left(W \rightarrow \tau \nu, Z \rightarrow \ell^{+} \ell^{-}\right)$. We use the NLO cross section $\sigma(W Z)=51.82 \mathrm{pb}$, according to [67]. We obtain: $\sigma_{\text {initial }}=$ $\sigma(W Z) \times\left(B R(W \rightarrow \ell \nu) B R\left(Z \rightarrow \tau^{+} \tau^{-}\right)+B R(W \rightarrow \tau \nu) B R\left(Z \rightarrow \ell^{+} \ell^{-}\right)\right)=(51.82 \times$ $\left.10^{3}\right) \times\left(0.22 \times 3.37 \times 10^{-2}+0.11 \times\left(6.7 \times 10^{-2}\right)\right) \mathrm{pb} \simeq 767 \mathrm{fb}$.

- $W+$ jets: the AlpGen tree-level cross section merged to the HERWIG ++ parton shower is $\sigma(W+$ jets $) \simeq 300 \mathrm{pb}$ per lepton flavour (electrons, muons or taus). This was calculated for 2 associated partons with the $W$ boson.

- $h V$ : the processes $h W$ and $h Z$ are included. We assume $\sigma(h W)=1504 \mathrm{fb}$ and $\sigma(h Z)=883 \mathrm{fb}$ at NNLO QCD + NLO EW, taken from [68] for $M_{h}=125 \mathrm{GeV}$. 
We allow $h \rightarrow W^{+} W^{-}$and $h \rightarrow \tau^{+} \tau^{-}$, impose no constrain on the $W$ decays and allow for the $Z$ to decay to all leptons. Hence: $\sigma_{\text {initial }}=\left(B R\left(h \rightarrow \tau^{+} \tau^{-}\right)+B R(h \rightarrow\right.$ $\left.\left.W^{+} W^{-}\right)\right) \times\left(\sigma(h Z) \times B R\left(Z \rightarrow \tau^{+} \tau^{-} / \ell^{+} \ell^{-}\right)+\sigma(h W)\right)=(0.0632+0.2155) \times(883 \times$ $\left.\left(10.1 \times 10^{-2}\right)+1504\right) \mathrm{fb} \simeq 443 \mathrm{fb}$.

- $t \bar{t} h, t \bar{t}$ : we assume that the efficiency of tagging jets originating from the decays $b$ quarks is $70 \%$. If one then vetoes events that contain at least one $b$-tagged jet, then for events containing $t \bar{t}$, a $1-\left(0.7^{2}+2 \times 0.3 \times 0.7\right)=0.09$ rejection factor can be achieved. We consider only leptonic decays of the $W$ bosons originating from the decays of the top quarks and only consider $h \rightarrow \tau^{+} \tau^{-}$. We assume total cross sections: $\sigma(t \bar{t}) \sim 900 \mathrm{pb}$ and $\sigma(t \bar{t} h) \simeq 611 \mathrm{fb}[68]$. This gives: $\sigma_{\text {initial }}(t \bar{t} \rightarrow$ leptons + jets $)=$ $0.09 \times 900 \mathrm{pb} \times B R(W \rightarrow \ell / \tau \nu)^{2} \simeq 8600 \mathrm{fb}$ and $\sigma_{\text {initial }}(t \bar{t} h \rightarrow$ leptons + jets + $\left.\left(\tau^{+} \tau^{-}\right)\right) \simeq 3.5 \mathrm{fb}$.

- $Z+$ jets: the AlpGen tree-level cross section merged to the HERWIG ++ parton shower is $\sigma(Z+$ jets $) \simeq 300 \mathrm{pb}$ per lepton flavour (electrons, muons or taus). This sample has been produced with one associated parton with the $Z$ boson.

\section{Discovery with low statistics}

Discovery occurs when the probability of obtaining a given experimental result, which contains some signal, is small when compared to the expected background hypothesis. How small this probability should be is somewhat a matter of preference and convention. Nowadays, in high energy physics, these probabilities are taken to correspond to 3 standard deviations away from the assumed central value of a Gaussian for the case of 'evidence' of a signal, and 5 standard deviations for the case of 'discovery' of a signal. On the other hand, exclusion is based on the probability of having fewer events than the background alone would give, given the signal plus background hypothesis.

To be concrete, let us assume that we are performing counting experiments of events, obtaining as a result, $N_{i}$ counts in each experiment $i$. Let us assume that in one specific experiment, we obtained a measurement $n_{\text {obs }}$. By some theoretical prediction, for example obtained using a Monte Carlo event generator, or otherwise, the expected background number of events in this experiment is given to be $b$. We can assume that the counts $N_{i}$ are random variables, distributed according to some distribution $P\left(N_{i}, b\right)$, where $b$ is the mean of the distribution. In this case, the probability of obtaining $n_{\text {obs }}$ or more events, when the mean is equal to the expected background $b$ is given by:

$$
P\left(N \geq n_{\mathrm{obs}}, b\right)=\sum_{i=n_{\mathrm{obs}}}^{i=\infty} P\left(N_{i}, b\right),
$$

where the sum can also be turned into an integral in the continuous variable case. In simple words, according to the 'background only' distribution, getting a measurement of $n_{\text {obs }}$ or more amounts to the probability of the shaded area in figure 13., and this probability tells you how likely $b$ is as an assumption of the mean of the distribution. 


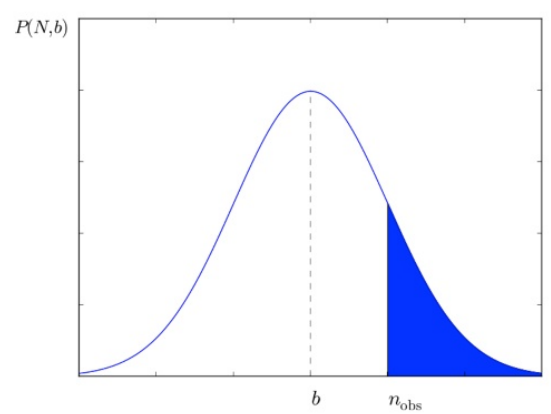

Figure 13. The shaded region in the above probability distribution shows the probability of obtaining $N>n_{\text {obs }}$ events.

In the specific case of the Poisson distribution:

$$
\operatorname{Pois}\left(N_{i}, b\right)=\frac{b^{N_{i}}}{N_{i} !} e^{-b}
$$

then eq. (D.1) becomes $P\left(N \geq n_{\mathrm{obs}}, b\right)=\sum_{i=n_{\mathrm{obs}}}^{i=\infty} \frac{b^{N_{i}}}{N_{i} !} e^{-b}$. This sum can be shown (see appendix E) to be equivalent to the so-called 'regularised incomplete gamma function', $\Gamma_{\text {reg }}(s, x)$ :

$$
P\left(N \geq n_{\mathrm{obs}}, b\right)=\sum_{i=n_{\mathrm{obs}}}^{i=\infty} \frac{b_{i}^{N}}{N_{i} !} e^{-b}=\Gamma_{\mathrm{reg}}\left(n_{\mathrm{obs}}, b\right)=\Gamma\left(n_{\mathrm{obs}}, b\right) / \Gamma\left(n_{\mathrm{obs}}\right),
$$

for $n_{\text {obs }}>0$, and where we have defined the 'unregularised incomplete gamma function':

$$
\Gamma\left(n_{\mathrm{obs}}, b\right)=\int_{0}^{b} \mathrm{~d} t t^{n_{\mathrm{obs}}-1} e^{-t},
$$

and $\Gamma\left(n_{\text {obs }}\right)$ is defined in eq. (E.2) in the following section, for $n=n_{\text {obs. }}$. We can then calculate the probability for discovery. This is given by $P\left(N \geq n_{\text {obs }}, b\right)$ for $n_{\text {obs }}=s+b$, where $s$ is the expected signal contribution to the event counts. This probability will differ from the one obtained using the large sample (i.e. Gaussian) approximation, in which the significance is given approximately by $\sigma \sim s / \sqrt{b}$. For exclusion, we need to calculate the probability of having less than $b$ events, under the assumption that the expected number of events is $s+b$, i.e. $P(N<b, s+b)$.

\section{E Cumulative distribution for Poisson random variables}

The unregularised incomplete gamma function is given by:

$$
\Gamma(n, x)=\int_{0}^{x} \mathrm{~d} t t^{n-1} e^{-t} .
$$

One can then define the gamma function:

$$
\Gamma(n)=\lim _{x \rightarrow \infty} \Gamma(n, x)=\int_{0}^{\infty} \mathrm{d} t t^{n-1} e^{-t} .
$$


The integral that appears in $\Gamma(n, x)$ can be expanded by performing consecutive integrations by parts:

$$
\begin{aligned}
\int_{0}^{x} \mathrm{~d} t t^{n-1} e^{-t}= & -\left.e^{-t} t^{n-1}\right|_{0} ^{x}+(n-1) \int_{0}^{x} \mathrm{~d} t t^{n-2} e^{-t} \\
= & -e^{-x} x^{n-1}-(n-1) e^{-x} x^{n-2} \\
& +(n-1)(n-2) \int_{0}^{x} \mathrm{~d} t t^{n-3} e^{-t} \\
= & -e^{-x}\left[x^{n-1}+(n-1) x^{n-2}\right. \\
& \left.+(n-1)(n-2) x^{n-3}+\ldots\right] \\
& +(n-1)(n-2)(n-3) \ldots(1)\left[1-e^{-x}\right] .
\end{aligned}
$$

From the last equality in the above expression we can deduce that

$$
\Gamma(n)=(n-1) ! .
$$

For $n>0$, dividing $\Gamma(n, x)$ by $\Gamma(n)$, we obtain:

$$
\begin{aligned}
\frac{\Gamma(n, x)}{\Gamma(n)} & =1-e^{-x}\left[\frac{x^{n-1}}{(n-1) !}+\frac{x^{n-2}}{(n-2) !}+\frac{x^{n-3}}{(n-3) !}+\ldots\right] \\
& =1-\sum_{i \leq n-1} \frac{x^{i} e^{-x}}{i !}=\sum_{i=n}^{\infty} \frac{x^{i} e^{-x}}{i !}
\end{aligned}
$$

which is nothing but the cumulative sum for the Poisson distribution.

\section{F Individual signal regions}

In figures 14 and 15 we demonstrate the individual signal regions contributing to the envelops shown in figures 8 and 9. The analyses at each luminosity are identical and the more 'irregular' form at lower luminosities is related to the Poisson statistics that govern the smaller number of events in those cases.

The sensitivity of the BSM searches depends on the hardness of the final state particles but not strongly on the angular distributions and polarisation. The hardness of the final state particles is primarily determined by the topology and mass spectrum of the BSM particles appearing in the process. Therefore, for example, if the LSP neutralino is replaced by a gravitino with the same mass, keeping the rest of the topology unchanged, the signal region efficiency is almost unchanged [69].

In table 6 we show the resulting cross sections after applying each of the signal regions, defined in table 5. These can be used in conjunction with the efficiency data files for the signal on the $M_{2}-M_{1}$ plane attached to this article ${ }^{13}$ to construct the signal cross section for each signal region for explicit BSM scenarios with $\tilde{N} \tilde{C}^{ \pm} \rightarrow(h \chi)\left(W^{ \pm} \chi\right)$ topology, where $\tilde{N}$

\footnotetext{
${ }^{13}$ The file corresponding to signal region $\mathrm{X}$ is "efficiency_regionX_expanded.dat", located in the subdirectory "efficiencies" of the distribution of this article.
} 

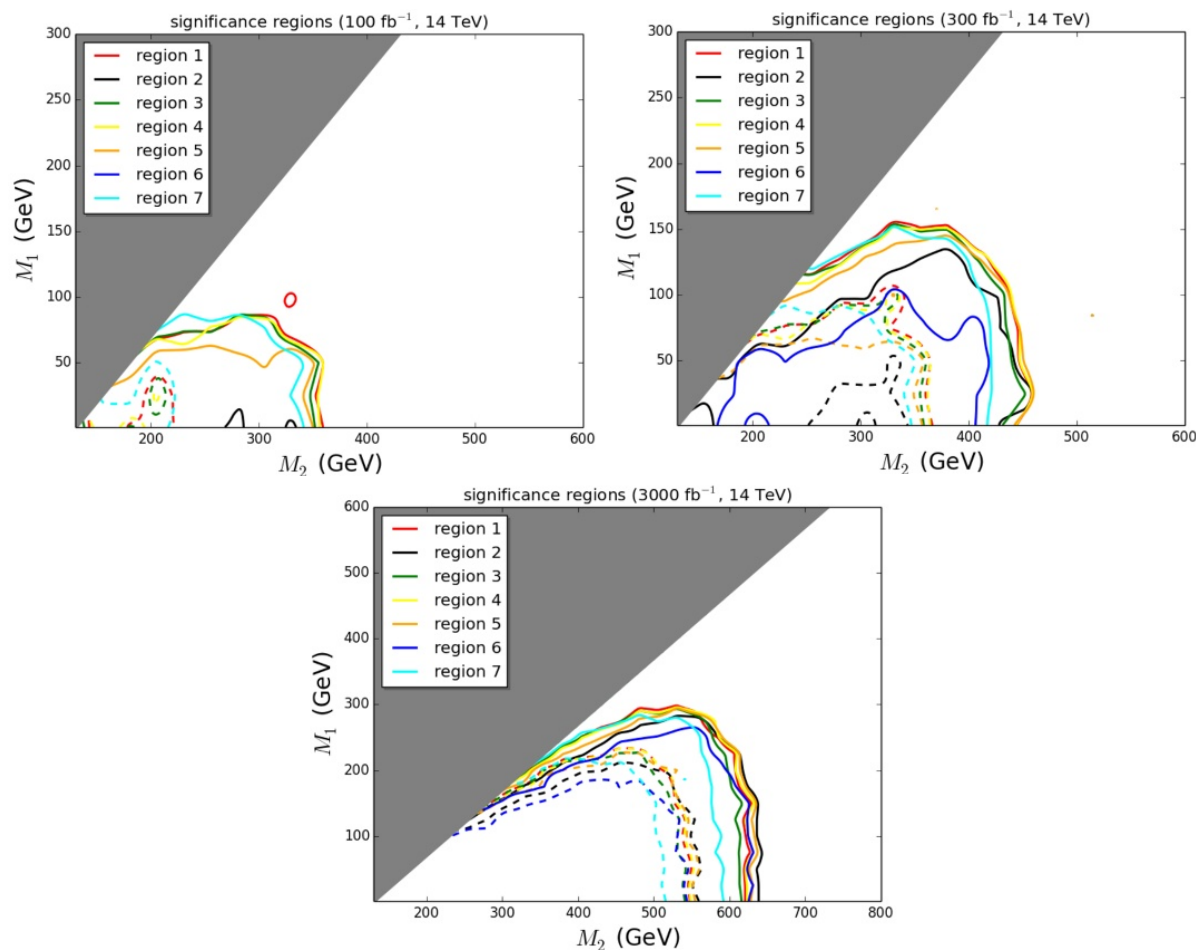

Figure 14. The significance on the $M_{2}-M_{1}$ plane obtained for the signal regions defined in table 5 at integrated luminosities of $100 \mathrm{fb}^{-1}$ (upper left), $300 \mathrm{fb}^{-1}$ (upper right) and $3000 \mathrm{fb}^{-1}$ (bottom). The solid curves show the $3 \sigma$ evidence region, whereas the dashed curves show the $5 \sigma$ discovery region.

\begin{tabular}{|llllllll|}
\hline signal region & SR1 & SR2 & SR3 & SR4 & SR5 & SR6 & SR7 \\
\hline$\sigma_{\text {bkg }}(\mathrm{fb})$ & 0.312 & 0.189 & 0.338 & 0.297 & 0.254 & 0.195 & 0.512 \\
\hline
\end{tabular}

Table 6. The resulting sum of cross sections for the backgrounds for the different signal regions (SR) used in the analysis.

and $\tilde{C}^{ \pm}$are massive BSM particles with the same mass, $M_{2}$, and $\chi$ is an invisible particle with mass $M_{1}$. This feature makes simplified models very useful for recasting analysis One can calculate the signal cross section for the process in question according to the given model:

$$
\text { [signal efficiency, signal region } \mathrm{X}] \times[\text { signal cross section }] \times[\mathrm{BR}],
$$

and use this in conjunction with the background cross section for region $\mathrm{X}$ as given in the table to obtain the p-value over the parameter space. Our efficiency data considers only the process with the $W \rightarrow \ell / \tau, \nu$ and Higgs bosons decaying inclusively to leptons (either $h \rightarrow \tau^{+} \tau^{-}$or $\left.h \rightarrow W^{+} W^{-} \rightarrow\left(e^{+} e^{-}, \mu^{+} \mu^{-}, \tau^{+} \tau^{-}\right)+\mathbb{E}_{T}\right)$. The [BR] factor in eq. (F.1) should therefore include these branching ratios. 

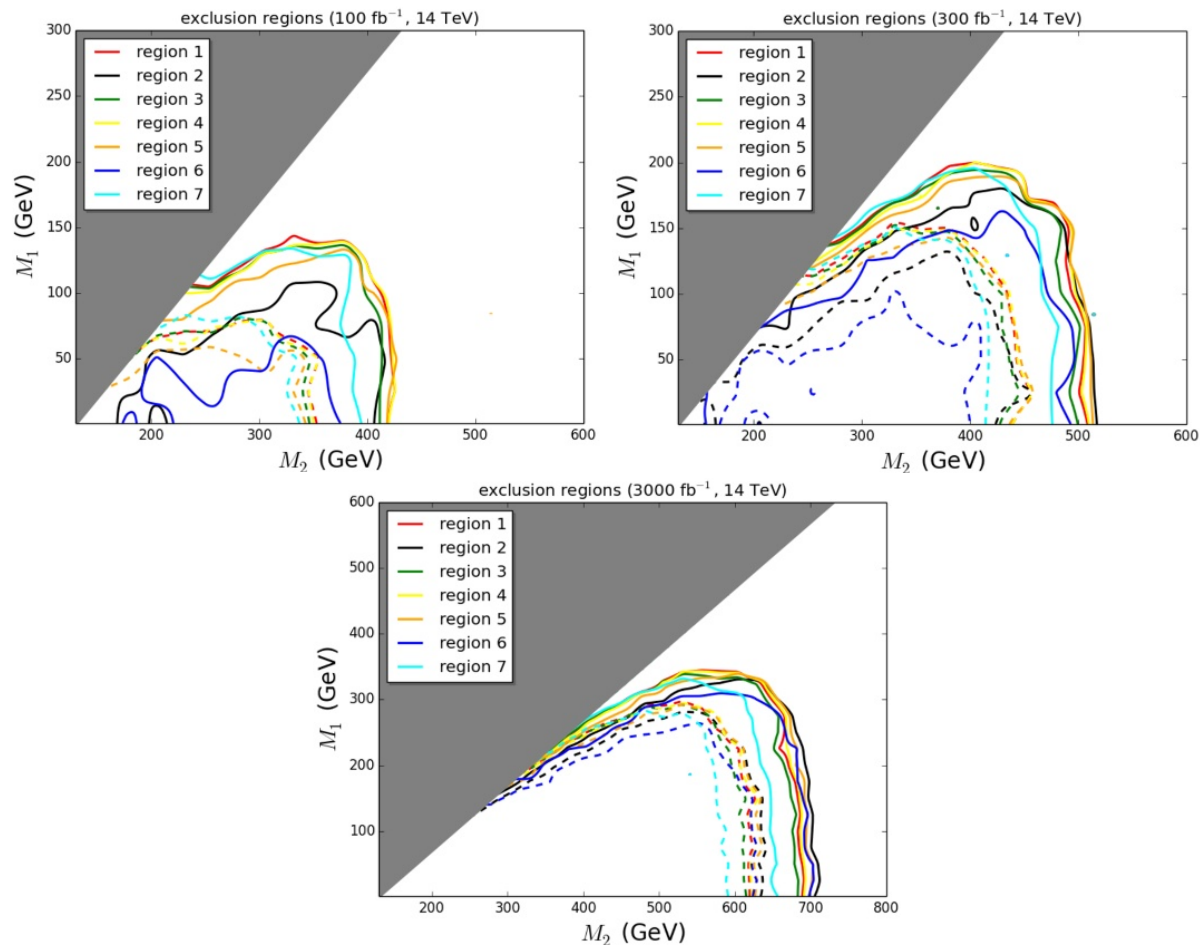

Figure 15. The exclusion on the $M_{2}-M_{1}$ plane obtained for the signal regions defined in table 5 at integrated luminosities of $100 \mathrm{fb}^{-1}$ (upper left), $300 \mathrm{fb}^{-1}$ (upper right) and $3000 \mathrm{fb}^{-1}$ (bottom). The solid curves show the $2 \sigma$ exclusion boundary, whereas the dashed curves show the $3 \sigma$ boundary.

\section{Acknowledgments}

We would like to thank Jonas Lindert for interesting comments and discussion. AP acknowledges support in part by the Swiss National Science Foundation (SNF) under contract 200020-149517, by the European Commission through the "LHCPhenoNet" Initial Training Network PITN-GA-2010-264564 and MCnetITN FP7 Marie Curie Initial Training Network PITN-GA-2012-315877. KS was supported in part by the London Centre for Terauniverse Studies (LCTS), using funding from the European Research Council via the Advanced Investigator Grant 267352. MT is grateful for funding from the Science and Technology Facilities Council (STFC).

Open Access. This article is distributed under the terms of the Creative Commons Attribution License (CC-BY 4.0), which permits any use, distribution and reproduction in any medium, provided the original author(s) and source are credited.

\section{References}

[1] ATLAS collaboration, Search for squarks and gluinos with the ATLAS detector in final states with jets and missing transverse momentum and $20.3 \mathrm{fb}^{-1}$ of $\sqrt{s}=8$ TeV proton-proton collision data, ATLAS-CONF-2013-047 (2013). 
[2] ATLAS collaboration, Search for squarks and gluinos in events with isolated leptons, jets and missing transverse momentum at $\sqrt{s}=8 \mathrm{TeV}$ with the ATLAS detector,

ATLAS-CONF-2013-062 (2013).

[3] ATLAS collaboration, Search for strongly produced supersymmetric particles in decays with two leptons at $\sqrt{s}=8 \mathrm{TeV}$, ATLAS-CONF-2013-089 (2013).

[4] ATLAS collaboration, Search for new phenomena in final states with large jet multiplicities and missing transverse momentum at $\sqrt{s}=8 \mathrm{TeV}$ proton-proton collisions using the ATLAS experiment, JHEP 10 (2013) 130 [arXiv:1308.1841] [INSPIRE].

[5] CMS Collaboration, Search for supersymmetry using razor variables in events with b-jets in pp collisions at $8 \mathrm{TeV}$, CMS-PAS-SUS-13-004 (2013).

[6] CMS Collaboration, Search for supersymmetry in hadronic final states using MT2 with the CMS detector at $\sqrt{s}=8 \mathrm{TeV}$, CMS-PAS-SUS-13-019.

[7] CMS collaboration, Search for new physics in the multijet and missing transverse momentum final state in proton-proton collisions at $\sqrt{s}=8 \mathrm{TeV}$, JHEP 06 (2014) 055 [arXiv: 1402.4770] [INSPIRE].

[8] M. Papucci, K. Sakurai, A. Weiler and L. Zeune, Fastlim: a fast LHC limit calculator, arXiv: 1402.0492 [INSPIRE].

[9] S. Kraml et al., SModelS: a tool for interpreting simplified-model results from the LHC and its application to supersymmetry, Eur. Phys. J. C 74 (2014) 2868 [arXiv:1312.4175] [INSPIRE].

[10] E. Conte, B. Fuks and G. Serret, MadAnalysis 5, A User-Friendly Framework for Collider Phenomenology, Comput. Phys. Commun. 184 (2013) 222 [arXiv:1206.1599] [INSPIRE].

[11] M. Drees, H. Dreiner, D. Schmeier, J. Tattersall and J.S. Kim, CheckMATE: Confronting your Favourite New Physics Model with LHC Data, arXiv:1312.2591 [INSPIRE].

[12] ATLAS collaboration, Observation of a new particle in the search for the Standard Model Higgs boson with the ATLAS detector at the LHC, Phys. Lett. B 716 (2012) 1 [arXiv: 1207.7214] [INSPIRE].

[13] CMS collaboration, Observation of a new boson at a mass of $125 \mathrm{GeV}$ with the CMS experiment at the LHC, CMS-HIG-12-028 (2012).

[14] G.F. Giudice and A. Strumia, Probing High-Scale and Split Supersymmetry with Higgs Mass Measurements, Nucl. Phys. B 858 (2012) 63 [arXiv:1108.6077] [INSPIRE].

[15] G. Kane, P. Kumar, R. Lu and B. Zheng, Higgs Mass Prediction for Realistic String/M Theory Vacua, Phys. Rev. D 85 (2012) 075026 [arXiv:1112.1059] [INSPIRE].

[16] M. Ibe and T.T. Yanagida, The Lightest Higgs Boson Mass in Pure Gravity Mediation Model, Phys. Lett. B 709 (2012) 374 [arXiv:1112.2462] [inSPIRE].

[17] A. Djouadi and J. Quevillon, The MSSM Higgs sector at a high $M_{\mathrm{SUSY}}$ : reopening the low $\tan \beta$ regime and heavy Higgs searches, JHEP 10 (2013) 028 [arXiv:1304.1787] [INSPIRE].

[18] H. Baer, V. Barger, A. Lessa, W. Sreethawong and X. Tata, Wh plus missing- $E_{T}$ signature from gaugino pair production at the LHC, Phys. Rev. D 85 (2012) 055022 [arXiv: 1201.2949] [INSPIRE].

[19] G.F. Giudice, M.A. Luty, H. Murayama and R. Rattazzi, Gaugino mass without singlets, JHEP 12 (1998) 027 [hep-ph/9810442] [INSPIRE]. 
[20] N. Arkani-Hamed and S. Dimopoulos, Supersymmetric unification without low energy supersymmetry and signatures for fine-tuning at the LHC, JHEP 06 (2005) 073 [hep-th/0405159] [INSPIRE].

[21] B.S. Acharya et al., Non-thermal Dark Matter and the Moduli Problem in String Frameworks, JHEP 06 (2008) 064 [arXiv:0804.0863] [INSPIRE].

[22] A. Arvanitaki, N. Craig, S. Dimopoulos and G. Villadoro, Mini-Split, JHEP 02 (2013) 126 [arXiv: 1210.0555] [INSPIRE].

[23] L.J. Hall and Y. Nomura, Spread Supersymmetry, JHEP 01 (2012) 082 [arXiv:1111.4519] [INSPIRE].

[24] M. Ibe, S. Matsumoto and T.T. Yanagida, Pure Gravity Mediation with $m_{3 / 2}=10-100 \mathrm{TeV}$, Phys. Rev. D 85 (2012) 095011 [arXiv:1202.2253] [InSPIRE].

[25] J.D. Wells, Implications of supersymmetry breaking with a little hierarchy between gauginos and scalars, hep-ph/0306127 [INSPIRE].

[26] B. Bhattacherjee, B. Feldstein, M. Ibe, S. Matsumoto and T.T. Yanagida, Pure Gravity Mediation of Supersymmetry Breaking at the LHC, Phys. Rev. D 87 (2013) 015028 [arXiv: 1207.5453] [INSPIRE].

[27] L.J. Hall, Y. Nomura and S. Shirai, Spread Supersymmetry with Wino LSP: Gluino and Dark Matter Signals, JHEP 01 (2013) 036 [arXiv:1210.2395] [INSPIRE].

[28] N. Arkani-Hamed, A. Gupta, D.E. Kaplan, N. Weiner and T. Zorawski, Simply Unnatural Supersymmetry, arXiv:1212.6971 [INSPIRE].

[29] ATLAS collaboration, Search for direct production of charginos and neutralinos in events with three leptons and missing transverse momentum in $\sqrt{s}=8 \mathrm{TeV}$ pp collisions with the ATLAS detector, JHEP 04 (2014) 169 [arXiv: 1402.7029] [INSPIRE].

[30] CMS Collaboration, Search for electroweak production of charginos, neutralinos and sleptons using leptonic final states in pp collisions at $8 \mathrm{TeV}$, CMS-PAS-SUS-13-006.

[31] CMS Collaboration, Search for electroweak production of charginos and neutralinos in final states with a Higgs boson in pp collisions at $8 \mathrm{TeV}$, CMS-PAS-SUS-13-017.

[32] ATLAS collaboration, Search for chargino and neutralino production in final states with one lepton, two b-jets consistent with a Higgs boson and missing transverse momentum with the ATLAS detector in 20.3 $\mathrm{fb}^{-1}$ of $\sqrt{s}=8 \mathrm{TeV}$ pp collisions, ATLAS-CONF-2013-093 (2013).

[33] T. Han, S. Padhi and S. Su, Electroweakinos in the Light of the Higgs Boson, Phys. Rev. D 88 (2013) 115010 [arXiv: 1309.5966] [INSPIRE].

[34] D. Ghosh, M. Guchait and D. Sengupta, Higgs Signal in Chargino-Neutralino Production at the LHC, Eur. Phys. J. C 72 (2012) 2141 [arXiv:1202.4937] [INSPIRE].

[35] P. Byakti and D. Ghosh, Magic Messengers in Gauge Mediation and signal for $125 \mathrm{GeV}$ boosted Higgs boson, Phys. Rev. D 86 (2012) 095027 [arXiv:1204.0415] [INSPIRE].

[36] A. Bharucha, S. Heinemeyer and F. von der Pahlen, Does the LHC exclude SUSY Particles at the ILC?, arXiv: 1404.0365 [INSPIRE].

[37] M. Asano, H.D. Kim, R. Kitano and Y. Shimizu, Natural Supersymmetry at the LHC, JHEP 12 (2010) 019 [arXiv: 1010.0692] [InSPIRE].

[38] Y. Kats, P. Meade, M. Reece and D. Shih, The Status of GMSB After 1/fb at the LHC, JHEP 02 (2012) 115 [arXiv:1110.6444] [inSPIRE]. 
[39] D. Curtin, P. Jaiswal and P. Meade, Charginos Hiding In Plain Sight, Phys. Rev. D 87 (2013) 031701 [arXiv: 1206.6888] [INSPIRE].

[40] K.-i. Hikasa, T. Liu, L. Wang and J.M. Yang, Pseudo-goldstino and electroweak gauginos at the LHC, JHEP 07 (2014) 065 [arXiv: 1403.5731] [INSPIRE].

[41] S.P. Martin, A supersymmetry primer, Adv. Ser. Direct. High Energy Phys. 21 (2010) 1 [hep-ph/9709356] [INSPIRE].

[42] W. Beenakker, R. Hopker and M. Spira, PROSPINO: A program for the production of supersymmetric particles in next-to-leading order QCD, hep-ph/9611232 [INSPIRE].

[43] W. Beenakker et al., The Production of charginos/neutralinos and sleptons at hadron colliders, Phys. Rev. Lett. 83 (1999) 3780 [Erratum ibid. 100 (2008) 029901] [hep-ph/9906298] [INSPIRE].

[44] A. Djouadi, Y. Mambrini and M. Muhlleitner, Chargino and neutralino decays revisited, Eur. Phys. J. C 20 (2001) 563 [hep-ph/0104115] [INSPIRE].

[45] A. Bharucha, S. Heinemeyer and F. von der Pahlen, Direct Chargino-Neutralino Production at the LHC: Interpreting the Exclusion Limits in the Complex MSSM, Eur. Phys. J. C 73 (2013) 2629 [arXiv: 1307.4237] [inSPIRE].

[46] A. Djouadi, M.M. Muhlleitner and M. Spira, Decays of supersymmetric particles: The Program SUSY-HIT (SUspect-SdecaY-HDECAY-InTerface), Acta Phys. Polon. B 38 (2007) 635 [hep-ph/0609292] [INSPIRE].

[47] M. Bahr et al., HERWIG++ Physics and Manual, Eur. Phys. J. C 58 (2008) 639 [arXiv: 0803.0883] [INSPIRE].

[48] K. Arnold et al., HERWIG++2.6 Release Note, arXiv:1205.4902 [INSPIRE].

[49] J. Bellm et al., HERWIG++ 2.7 Release Note, arXiv:1310.6877 [INSPIRE].

[50] M.L. Mangano, M. Moretti and R. Pittau, Multijet matrix elements and shower evolution in hadronic collisions: $W b \bar{b}+n$ jets as a case study, Nucl. Phys. B 632 (2002) 343 [hep-ph/0108069] [INSPIRE].

[51] M. Mangano, Merging multijet matrix elements and shower evolution in hadronic collisions, http://mlm.web.cern.ch/mlm/talks/lund-alpgen.pdf (2004).

[52] J. Alwall et al., Comparative study of various algorithms for the merging of parton showers and matrix elements in hadronic collisions, Eur. Phys. J. C 53 (2008) 473 [arXiv:0706.2569] [INSPIRE].

[53] M. Bahr, S. Gieseke and M.H. Seymour, Simulation of multiple partonic interactions in HERWIG++, JHEP 07 (2008) 076 [arXiv:0803.3633] [INSPIRE].

[54] B. Gripaios, K. Nagao, M. Nojiri, K. Sakurai and B. Webber, Reconstruction of Higgs bosons in the di-tau channel via 3-prong decay, JHEP 03 (2013) 106 [arXiv:1210.1938] [INSPIRE].

[55] ATLAS collaboration, Identification of the Hadronic Decays of Tau Leptons in 2012 Data with the ATLAS Detector, ATLAS-CONF-2013-064 (2013).

[56] CMS collaboration, CMS Strategies for tau reconstruction and identification using particle-flow techniques, CMS-PAS-PFT-08-001.

[57] A. Katz, M. Son and B. Tweedie, Ditau-Jet Tagging and Boosted Higgses from a Multi-TeV Resonance, Phys. Rev. D 83 (2011) 114033 [arXiv:1011.4523] [INSPIRE]. 
[58] C. Englert, T.S. Roy and M. Spannowsky, Ditau jets in Higgs searches, Phys. Rev. D 84 (2011) 075026 [arXiv: 1106 .4545] [INSPIRE].

[59] M. Cacciari, G.P. Salam and G. Soyez, FastJet User Manual, Eur. Phys. J. C 72 (2012) 1896 [arXiv: 1111.6097] [INSPIRE].

[60] ATLAS collaboration, Performance of the Reconstruction and Identification of Hadronic Tau Decays with ATLAS, ATLAS-CONF-2011-152 (2011).

[61] ATLAS collaboration, Expected Performance of the ATLAS Experiment - Detector, Trigger and Physics, arXiv:0901.0512 [INSPIRE].

[62] ATLAS collaboration, Performance assumptions for an upgraded ATLAS detector at a High-Luminosity LHC, ATL-PHYS-PUB-2013-004 (2013).

[63] ATLAS collaboration, Physics at a High-Luminosity LHC with ATLAS, ATL-PHYS-PUB-2013-007 (2013).

[64] ATLAS collaboration, Search for Supersymmetry at the high luminosity LHC with the ATLAS experiment, ATL-PHYS-PUB-2014-010 (2014).

[65] B. Gripaios, A. Papaefstathiou, K. Sakurai and B. Webber, Searching for third-generation composite leptoquarks at the LHC, JHEP 01 (2011) 156 [arXiv:1010.3962] [INSPIRE].

[66] B. Gripaios, K. Sakurai and B. Webber, Polynomials, Riemann surfaces and reconstructing missing-energy events, JHEP 09 (2011) 140 [arXiv:1103.3438] [INSPIRE].

[67] J.M. Campbell, R.K. Ellis and C. Williams, Vector boson pair production at the LHC, JHEP 07 (2011) 018 [arXiv: 1105.0020] [INSPIRE].

[68] LHC Higgs Cross section Working Group collaboration, S. Dittmaier et al., Handbook of LHC Higgs Cross sections: 1. Inclusive Observables, arXiv:1101.0593 [INSPIRE].

[69] LHC New Physics Working Group collaboration, D. Alves et al., Simplified Models for LHC New Physics Searches, J. Phys. G 39 (2012) 105005 [arXiv:1105.2838] [InSPIRE]. 